\title{
The dimerization domain of PfCENP-C is required for its functions as a centromere protein in human malaria parasite Plasmodium falciparum
}

\author{
Garima Verma and Namita Surolia*
}

\begin{abstract}
Background: The conserved centromere-associated proteins, CENH3 (or CENP-A) and CENP-C are indispensable for the functional centromere-kinetochore assembly, chromosome segregation, cell cycle progression, and viability. The presence and functions of centromere proteins in Plasmodium falciparum are not well studied. Identification of PFCENP-C, an inner kinetochore protein (the homologue of human CENP-C) and its co-localization with PFCENH3 was recently reported. This study aims to decipher the functions of inner kinetochore protein, PfCENP-C as a centromere protein in P. falciparum.

Methods: Bio-informatic tools were employed to demarcate the two conserved domains of PFCENP-C, and the functions of PfCENP-C domains were demonstrated by functional complementation assays in the temperature sensitive (TS) mutant strains (mif2-3 and mif2-2) of Saccharomyces cerevisiae with MIF2p (the yeast homologue of CENP-C) loss-of-function. By site-directed mutagenesis, the key residues essential for PfCENP-C functions were determined. The chromatin immunoprecipitation was carried out to determine the in vivo binding of PfCENP-C to the Plasmodium centromeres and the in vivo interactions of PFCENP-C with PFCENH3, and mitotic spindles were shown by co-immunopreciptation experiments.

Results: The studies demonstrate that the motif and the dimerization domain of PFCENP-C is able to functionally complement MIF2p functions. The essential role of some of the key residues: F1993, F1996 and Y2069 within the PFCENP-C dimerization domain in mediating its functions and maintenance of mitotic spindle integrity is evident from this study. The pull-down assays show the association of PfCENP-C with PfCENH3 and mitotic spindles. The ChIP-PCR experiments confirm PFCENP-C-enriched Plasmodium centromeres. These studies thus provide an insight into the roles of this inner kinetochore protein and establish that the centromere proteins are evolutionary conserved in the parasite.

Conclusions: PFCENP-C is a true CENP-C homologue in P. falciparum which binds to the centromeric DNA and its dimerization domain is essential for its in vivo functions as a centromere protein. The identification and functional characterization of the $P$. falciparum centromeric proteins will provide mechanistic insights into some of the mitotic events that occur during the chromosome segregation in human malaria parasite, $P$. falciparum.
\end{abstract}

Keywords: Plasmodium falciparum, PfCENP-C, Functional complementation, PfCENP-C motif, PfCENP-C dimerization domain

\footnotetext{
* Correspondence: surolia@jncasr.ac.in

Molecular Parasitology Laboratory, Molecular Biology and Genetics Unit,

Jawaharlal Nehru Centre For Advanced Scientific Research, Jakkur, Bangalore 560064 , India
} 


\section{Background}

During cell division, the fidelity in chromosome segregation is ensured by the specialized locus on the chromosome, the centromere. The kinetochore proteins assemble on the centromeric chromatin and mediate the attachment of the microtubule spindles to the chromosomes. In all the eukaryotes studied so far, CENP-A and CENP-C together form the core components of the centromeric chromatin and are indispensable for centromere formation and function. CENP-C is an integral part of the inner kinetochore plate and serves as an interface between the centromeric chromatin and the outer kinetochore plate to which spindle microtubules attach [1-5]. A functional kinetochore assembly depends on the recruitment of the CENP-C to the CENP-A-containing centromeric nucleosomes and the subsequent CENP-C-dependent recruitment of a subset of inner and outer kinetochore proteins on the centromere [6-12]. Like CENP-A, CENP-C constitutively localizes to the active centromeres and is required for the kinetochore assembly and timely progression during cell cycle [1,3,13-20]. Loss of CENP-C function results in abolishing the centromere-kinetochore assembly, chromosome mis-segregation, metaphase/anaphase block and cell death, suggesting its indispensable role in proper chromosome segregation and viability in the eukaryotes [2,4,13,18,21-26].

Detailed analyses of CENP-C revealed that it is composed of different domains, each with specialized functions [6,17,27-29]. It comprises of an amino terminal domain, a central region, a signature CENP-C motif, and a carboxy terminal dimerization domain. The amino terminal domain of CENP-C is shown to confer oligomerization and instability on CENP-C and is not required for centromere targeting $[27,30]$. This domain binds directly to the components of Mis12 kinetochore complex and prevents the excessive accumulation and mistargeting of CENP-C to the non-centromeric sites [4,20,27,30,31]. The central region of $\mathrm{CENP}-\mathrm{C}$ has been shown to carry a DNA-binding domain with an overlapping centromeretargeting domain $[3,17,27,28,32,33]$. The signature CENP$\mathrm{C}$ motif binds to the CENP-A-containing centromeric nucleosomes, centromeric $\alpha$-satellite DNA and is required for the centromere targeting [25,34-37]. The dimerization domain is responsible for the higher order structural organization of CENP-C, self-dimerization, centromeric targeting and in vivo kinetochore assembly $[6,10,27,28,30]$.

In Saccharomyces cerevisiae, the mutations in the MIF2p domains, namely, the CENP-C motif and the dimerization domain, lead to impaired centromere-kinetochore structure, metaphase to anaphase delay and chromosome missegregation $[6,25,36,37]$. Thus, this mitotic fidelity gene is shown to have an essential role in chromosome segregation and mitotic spindle integrity and the cells deficient in
MIF2p show abnormal phenotypes, defective spindle morphologies and delayed progression during mitosis $[21,36,38]$.

Recent studies on the centromere-specific $\mathrm{H} 3$ variant have contributed to the current understanding of centromere proteins in Plasmodium falciparum [39]. The Plasmodium centromeres are enriched with PfCENH3 and are important for centromere assembly and propagation [40]. The structural-functional determinants of PfCENH3 that confer centromere specific functions and the association of PfCENH3 with another putative inner kinetochore protein PfCENP-C have also been demonstrated in this parasite [39].

This study reports the identification of various CENP$\mathrm{C}$ domains and detailed functional dissection of its dimerization domain in $P$. falciparum. The two domains of PfCENP-C, namely, the CENP-C motif and the dimerization domain are mapped using bio-informatic analysis and their roles as a centromere protein are tested by the functional complementation of MIF2p. In budding yeast, the temperature-sensitive (TS) mutants, mif2-2 and mif2-3 harbour different mutations which results in MIF2p loss-of-function. The mif2-2 TS strain carries a 'S309L' mutation in the CENP-C motif as well as a 'S500F' mutation in its dimerization domain $[21,34,36]$, the mif2-3 TS strain has a 'P505L' mutation in the MIF2p dimerization domain (MIF2p ${ }^{\mathrm{P} 505 \mathrm{~L}}$ ) $[6,34]$ that confers the TS phenotype. While, the PfCENP-C dimerization domain could functionally substitute for the MIF2p loss-of-function in the mif2-3 TS mutant, it could partially complement the MIF2p loss-of-functions in mif2-2 TS mutants. The PfCENP-C motif show partial rescue in the mif2-3 as well as mif2-2 TS mutants, respectively. These TS yeast cells at non-permissive temperature show aberrant phenotype features such as chromosome mis-segregation and broken microtubules that are rescued upon the expression of PfCENP-C dimerization domain. The indispensable role of the PfCENP-C dimerization domain in mediating the centromere protein functions is confirmed by the mutational analysis of the conserved residues (F1993, F1996 and Y2069) within the PfCENP-C dimerization domain as compared to the conserved N2060 residue in the PfCENPC dimerization domain corresponding to the MIF2p N514 residue, which is not required for its functions. The PfCENP-C ${ }^{\mathrm{P} 2051 \mathrm{~L}}$ dimerization domain mutant corresponding to MIF2 $\mathrm{p}^{\mathrm{P} 505 \mathrm{~L}}$ TS mutation, as present in the mif2-3 TS mutant strain also fails to overcome the growth defects. Severe functional and phenotypic defects on growth, chromosome segregation and mitotic spindle structures are observed upon the expression of PfCENP-C dimerization domain mutants, demonstrating the importance of a functional dimerization domain in the kinetochore assembly, chromosome segregation and maintenance of the mitotic spindle integrity. 
In addition, ChIP-PCR data shows the enrichment of $P f C E N H 3$ and PfCENP-C on the centromeric regions of $P$. falciparum as compared to the non-centromeric regions. The immunoprecipitation experiments demonstrate the interactions of PfCENP-C with PfCENH3 and tubulin. Thus, the data presented here establishes the putative PfCENP-C as a true CENP-C homologue and the role of its dimerization domain in mediating the functions of PfCENP-C.

\section{Methods}

\section{Plasmodium falciparum culture}

The P. falciparum FCK2 (a local strain of Karnataka, India) was cultured in $\mathrm{O}^{+}$human erythrocytes, RPMI 1640 medium supplemented with 25 mM HEPES, 0.2\% $\mathrm{NaHCO}_{3}$ and $10 \%(\mathrm{v} / \mathrm{v})$ heat-inactivated $\mathrm{O}^{+}$human serum by standard candle jar method [41]. Tight synchronization of cultures was performed by $5 \% \mathrm{D}$-sorbitol [42] and the parasites were isolated by $0.15 \%(\mathrm{w} / \mathrm{v})$ saponin lysis followed by subsequent washes of the parasite pellet with cold phosphate-buffered saline pH7.4 (1× PBS).

\section{Bio-informatics analysis}

Using the BLASTp [43], the amino acid sequences of conserved CENP-C motif and the C-terminus dimerization domain of human CENP-C were used as queries to blast against the whole P. falciparum genome database [44]. Using ClustalW and Espript 3.0, the multiple sequence alignments were performed between PfCENP-C and its homologues. Percent pair-wise similarities between the full-length CENP-C homologues were determined by Needleman-Wunsch alignment.

\section{Antibodies}

The peptide sequence N- VEVILVEKKLKKKKQKC-C was used to generate PfCENP-C polyclonal antibodies in mice followed by affinity purification with the respective peptide (Genemed Synthesis, USA). To stain the mitotic spindles, mouse monoclonal anti $\alpha$-tubulin (Calbiochem, USA) was used. Monoclonal anti-GFP (Roche, USA) was used for Western blot and immunofluorescence assays.

\section{Western blot analysis}

The isolated parasites from various asexual stages were lysed in RIPA buffer $(150 \mathrm{mM} \mathrm{NaCl}, 50 \mathrm{mM}$ Tris- $\mathrm{HCl}$, $\mathrm{pH}$ 7.4, $1 \% \mathrm{NP}-40,0.25 \%$ sodium deoxycholate, $1 \mathrm{mM}$ EDTA, $1 \mathrm{mM}$ sodium orthovanadate, protease inhibitor cocktail) [45] and mechanically sheared by passing through 1-ml syringe. The standard Bradford method [46] was used to estimate the protein concentration of the lysates. The whole cell parasite extract was resolved on 6 and 12\% SDS polyacrylamide gel electrophoresis (Mini Protean Tetrad apparatus, Biorad, USA) followed by immunoblotting on PVDF membrane (Millipore,
India). The parasite extracts were probed using antiPfCENP-C (1:1,000), anti-GAPDH (1:2,000, Sigma-Aldrich, Germany), anti-PfCENH3 (1:2,000, Genemed Synthesis, USA) and anti- $\alpha$ tubulin (1:2,000, Calbiochem, USA) followed by incubation with goat anti-mouse and antirabbit secondary antibodies conjugated with horseradish peroxidase (1:3,000, Bangalore Genei, India). ECL substrate (Biorad, USA) was used to detect the signals of the Western analyses.

In S. cerevisiae, the Western blot analyses were carried out by growing the yeast strains expressing different constructs both at permissive and restrictive temperatures at $\mathrm{A}_{600}=\sim 1$. For lysate preparation, the yeast pellets were washed twice with autoclaved $\mathrm{H}_{2} \mathrm{O}$ and lysed in $50 \%$ TCA for $1 \mathrm{hr}$ at $-80^{\circ} \mathrm{C}$ followed by two washes with $80 \%$ acetone. The air-dried pellets were resuspended in $0.1 \% \mathrm{SDS} / 0.1 \mathrm{~N} \mathrm{NaOH}$. Western blot analyses were carried out on the lysates and the GFP-tagged PfCENP-C domains were detected by mouse monoclonal anti-GFP (1:3,000, Roche, USA).

\section{Yeast strains, expression constructs and site-directed mutagenesis}

For the functional complementation assays, two different TS strains deficient in MIF2p, Y06477 (mif2-3: KanR: his $3^{\wedge} 1$ leu $2^{\wedge} 0$ ura3 $^{\wedge} 0$ met $15^{\wedge} 0$ ); 6848-4-2 (Mat a ura3 mif2-2) [21,34,36], and the wild type (WT) strain DMA3865 (Mat a; ura10^Kan his $3^{\wedge} 1$ leu $2^{\wedge} 0$ ura $3^{\wedge} 0$ met $\left.15^{\wedge} 0\right)$ were used. YPDU-agar and YPDU broth $(1 \%$ yeast extract, $2 \%$ peptone, $2 \%$ dextrose and $0.01 \%$ uracil) were used to grow all the strains at $23^{\circ} \mathrm{C}$ (permissive temperature). The mif2-2 TS mutant strain has 'S309L' mutation in the CENP-C motif and a 'S500F' mutation in its dimerization domain $[21,34,36]$. The mif2-3 TS mutant strain has a 'P505L' point mutation in the MIF2p dimerization domain [6,34]. Both the TS mutant strains show growth arrest at $37^{\circ} \mathrm{C}$ (non-permissive temperature). The yeast expression vector $\mathrm{p} 415 \mathrm{ADH}$-containing ADH1 promoter, leucine selectable marker and the terminator sequences was used for cloning the various PfCENP-C domains between the $\mathrm{XbaI}$ and $\mathrm{BamHI}$ restriction enzyme sites. To clone the N-terminal region (NTD) of PfCENPC, first 540 nucleotides of full-length PfCENP-C was used. Based on the sequence comparisons, the nucleotide sequences spanning the PfCENP-C motif and the PfCENP-C dimerization domain were used for cloning these respective PfCENP-C domains in p415ADH. The full-length MIF2p from $S$. cerevisiae was also cloned into the $\mathrm{XbaI}$ and HindIII sites of p415ADH. The vector carrying PfCENP-C domains, full-length MIF2p and empty vector alone were transformed into the WT strain (DMA3865) and the TS mif2-3 and mif2-2 mutant yeast cells (Y06477 and 6848-42) by lithium acetate method [47]. Synthetic media devoid of leucine was used to select the transformants. The Quick 
Change II XL site-directed mutagenesis kit (Stratagene, USA) was used to substitute the conserved amino acid residues in the PfCENP-C dimerization domain following the protocol recommended by the manufacturer. The point mutations were incorporated by using the primers spanning the nucleotide coding for leucine and alanine for substitution of proline at 2051 position and F1993, F1996, N2060 and Y2069, respectively in the PfCENP-C dimerization domain. The expression construct containing the ORF (PfCENP-C dimerization domain) was used as a template (10-50 ng) and amplified with the high fidelity Pfu polymerase. The methylated template DNA was digested by Dpn I for $1 \mathrm{hr}$ at $37^{\circ} \mathrm{C}$ followed by transformation into $\mathrm{DH} 5 \alpha$ competent cells. The desired mutations were confirmed by sequencing and transformed into the mif2-3 and mif22 TS mutants (Y06477 and 6848-4-2) and the WT strain (DMA3865).

\section{Functional complementation assay}

To assess the complementation ability of PfCENP-C domains, the mif2-3 and mif2-2 transformants expressing PfCENP-C N-terminal region (NTD), PfCENP-C motif, PfCENP-C dimerization domain (DD), MIF2p and empty vector were streaked on the plates containing selective media devoid of leucine and fully supplemented YPDU agar, incubated at $23^{\circ} \mathrm{C}$ and $37^{\circ} \mathrm{C}$ for three days and imaged. For ten-fold spot dilution experiments, the yeast strains expressing the abovementioned expression constructs were grown in YPDU broth at $23^{\circ} \mathrm{C}$ to attain $\mathrm{A}_{600}=\sim 1$. Serial dilutions starting from $5 \times 10^{5} \mathrm{cells} \mathrm{ml}^{-1}$ to $5 \times 10^{-1}$ cells $\mathrm{ml}^{-1}$ were prepared and spotted on YPDU plates and allowed to grow at $23^{\circ} \mathrm{C}$ and $37^{\circ} \mathrm{C}$ for three days. The mif2-3 and mif2-2 TS mutant cells expressing full-length MIF2p from $S$. cerevisiae and the empty vector alone ( $\mathrm{p} 415 \mathrm{ADH})$ were used as positive and negative controls, respectively. The phenotypes of the various transformants were visualized and imaged by fixing the cells with $70 \%$ alcohol and Hoechst staining of the nuclei followed by confocal imaging. The gain-or loss-of-phenotypes was analysed and scored by imaging the bud morphologies and the nuclear staining.

\section{Indirect immunofluorescence assay}

The tightly synchronized parasite cultures were processed for indirect immunofluorescence assays as previously described [48]. The parasites were washed with PBS and fixed using $4 \%$ paraformaldehyde and $0.0075 \%$ glutaraldehyde in PBS (EM grade, Polysciences Inc, USA) $30 \mathrm{~min}$ at room temperature and for permeabilization $0.05 \%$ Triton $\mathrm{X}-100$ was used for $2 \mathrm{~min}$ at room temperature followed by blocking with $3 \% \mathrm{BSA}$ for $1 \mathrm{hr}$ at $4^{\circ} \mathrm{C}$. Both the primary and secondary antibodies were diluted in 3\% BSA and incubated for $1 \mathrm{hr}$ at room temperature. The primary antibody, mouse anti-PfCENP-C and the secondary antibody,
Alexa Fluor 568 conjugated goat anti-mouse were used at 1:100 and 1:200 (Molecular Probes, USA) dilutions, respectively. Hoechst3328 (Sigma-Aldrich, USA) was used to stain nucleus followed by mounting over the glass slides using Vectashield (Sigma-Aldrich, USA).

In $S$. cerevisiae, the indirect immunofluorescence assays were performed on the mif2-3 and mif2-2 TS mutant cells expressing various PfCENP-C domains, MIF2p and empty vector using protocol as previously described [47]. The cells were allowed to grow at $A_{600}=\sim 1$ and fixed with $37 \%$ formaldehyde $\left(1 / 10^{\text {th }}\right.$ culture volume) for $1 \mathrm{hr}$. Subsequently, the cells were washed with $0.1 \mathrm{M}$ phosphate buffer ( $\mathrm{pH}$ 6.4) and allowed to sphaeroplast using lyticase (Sigma-Aldrich, USA) and 2-mercapto ethanol at $30^{\circ} \mathrm{C}, 65 \mathrm{rpm}$. Teflon-coated slides incubated with poly-L-lysine ( $1 \mathrm{mg} \mathrm{ml}^{-1}$, Sigma-Aldrich, Germany) were used to adhere the cells. The cells on the slides were fixed in ice-cold methanol for $6 \mathrm{~min}$ at $-20^{\circ} \mathrm{C}$ and ice-cold acetone for $30 \mathrm{sec}$ at $-20^{\circ} \mathrm{C}$. The blocking was performed in a humid chamber with $2 \%$ skimmed milk in PBS for 30 min followed by incubation with anti- $\alpha$ tubulin (1:200) and anti-GFP (1:400) for $1 \mathrm{hr}$. The cells were then incubated with Alexafluor 488 secondary antibodies (1:200) for $1 \mathrm{hr}$. PBS washes were carried out between the two antibodies incubation and the nuclei were stained with Hoechst 3328 (Sigma-Aldrich, USA). All the confocal images were evenly adjusted for brightness and contrast.

\section{Chromatin immunoprecipitation (ChIP)}

The ChIP assays were performed as described by $[49,50]$. The parasite cultures were crosslinked by addition of $37 \%$ formaldehyde (Sigma-Aldrich, USA) to get a final concentration of $1 \%$ for $5 \mathrm{~min}$ at $37^{\circ} \mathrm{C}$. The crosslinking was stopped by the addition of $0.125 \mathrm{M}$ glycine for $5 \mathrm{~min}$ on ice. The crosslinked parasites were washed thrice in icecold PBS and $0.06 \%$ saponin was used for RBC lysis. After saponin lysis, the parasite pellet was again washed twice with $1 \times$ PBS ( $\mathrm{pH} 7.4)$. The isolated crosslinked parasites were resuspended in cold lysis buffer $(10 \mathrm{mM} \mathrm{KCl}$, 10 mM Hepes pH 7.9, 0.1 mM EGTA pH 8.0, $0.1 \mathrm{mM}$ EDTA pH 8.0, 1 mM DTT and protease inhibitor cocktail) and transferred to a douncer homogenizer for lysis with the addition of NonidetP-40 at a final concentration of $0.25 \%$. The lysate was centrifuged at $16,000 \times \mathrm{g}$ for $10 \mathrm{~min}$ at $4^{\circ} \mathrm{C}$. The pellet obtained was resuspended in SDS lysis buffer $(50 \mathrm{mM}$ Tris- $\mathrm{HCl} \mathrm{pH} 8.1,1 \%$ SDS and $10 \mathrm{mM}$ EDTA). To get sheared chromatin, sonication was performed at high power with $30 \mathrm{sec}$ on and offcyclings for $10 \mathrm{~min}$ (Diagenode Bioruptor). The sheared chromatin was collected by centrifugation at $12,500 \times \mathrm{g}$ for $10 \mathrm{~min}$ at $4^{\circ} \mathrm{C}$ and diluted ten-fold in ChIP dilution buffer $(1.1 \%$ Triton X-100, 0.01\% SDS, 16.7 mM Tris- $\mathrm{HCl} \mathrm{pH}$ 8.1, $150 \mathrm{mM} \mathrm{NaCl}$ and $1.2 \mathrm{mM}$ EDTA). For chromatin-IP 
assays, the chromatin solutions were precleared with protein A or G agarose slurry (GE Healthcare, Sweden) and $50 \mu \mathrm{l} \mathrm{mL}{ }^{-1}$ of salmon sperm DNA (Sigma-Aldrich, USA) for $2 \mathrm{hr}$ at $4^{\circ} \mathrm{C}$. The precleared chromatin solutions were incubated with PfCENP-C and PfCENH3 antibodies for $12 \mathrm{hr}$ at $4^{\circ} \mathrm{C}$. The immune complexes were collected by centrifugation at $1,000 \times \mathrm{g}$ for $1 \mathrm{~min}$ and washed with three different wash buffers at $4^{\circ} \mathrm{C}$, the low salt wash buffer (20 mM Tris- $\mathrm{HCl} \mathrm{pH}$ 8.1, 2 mM EDTA, 0.1\% SDS, $150 \mathrm{mM} \mathrm{NaCl}$ and $1 \%$ Triton X-100) followed by the high salt wash buffer (20 mM Tris-HCl pH 8.1, 2 mM EDTA, $0.1 \%$ SDS, $500 \mathrm{mM} \mathrm{NaCl}$ and $1 \%$ Triton X-100) and $\mathrm{LiCl}$ wash buffer (10 mM Tris- $\mathrm{HCl} \mathrm{pH} \mathrm{8.1,} 0.25 \mathrm{M} \mathrm{LiCl}, 1 \mathrm{mM}$ EDTA, 1\% NP-40 and 1\% deoxycholate). The final washes were performed with TE $(10 \mathrm{mM}$ Tris- $\mathrm{HCl} \mathrm{pH} 8.0$ and $1 \mathrm{mM}$ EDTA, pH 8.0) buffer at room temperature. The elution was performed with freshly prepared elution buffer (0.1 M NaHCO3 and 1\% SDS) at room temperature. To obtain DNA, the eluates and the input samples were decrosslinked by incubating at $65^{\circ} \mathrm{C}$ followed by treatment with Proteinase $\mathrm{K}$ and RNaseA. The DNA was purified by phenol/chloroform/isoamyl alcohol extraction method. The immunoprecipitated as well as the input DNA samples were quantified and used as templates for semiquantitative PCR reactions. To ensure that the amplifications are in linear range, the sub-saturation point was standardized to avoid end point analysis using Q-PCR amplification curves. For each set of primers, 5 cycles less than the point of saturation were performed and then the products were run on the gel for quantification. The fold enrichment (ChIP over input) for each centromeric as well as non-centromeric region was determined by quantifying the gel band intensities using AlphaImager.

\section{Immunoprecipitation}

The immunoprecipitation assays were performed using PfCENP-C and PfCENH3 antibodies as previously described by [45]. The parasites were isolated at late trophozite and schizont stages and lysed in modified RIPA buffer $(150 \mathrm{mM} \mathrm{NaCl}, 50 \mathrm{mM}$ Tris- $\mathrm{HCl}, \mathrm{pH}$ 7.4, 1\% NP40, $0.25 \%$ sodium deoxycholate, $1 \mathrm{mM}$ EDTA, $1 \mathrm{mM}$ sodium orthovanadate, protease inhibitor cocktail). Prior to IP, the lysates were precleared by incubating with Protein A or G agarose beads (GE Healthcare, Sweden) for $4 \mathrm{hr}$ at $4^{\circ} \mathrm{C}$ and the antibodies were conjugated with the agarose beads. The protein concentration was estimated by standard Bradford method [46] based on which the amount of antibody/IP was determined ( 10 ug of Ab for 5 ug of total protein). To immunoprecipitate the protein of interest, the total protein was incubated with the antibody-conjugated beads overnight at $4^{\circ} \mathrm{C}$. The immunocomplexes were collected by centrifugation followed by three washes with chilled lysis buffer. The immunoprecipitated protein was eluted by $0.1 \mathrm{M}$ glycine $\mathrm{pH} 3.4$ followed by the neutralization. The eluates were subjected to Western blot analyses as described above.

\section{Results \\ PfCENP-C has conserved CENP-C motif and dimerization domain}

The bio-informatic analyses have shown that CENP-C homologues display maximum sequence conservation at the CENP-C signature motif and the dimerization domain $[29,34,51]$. To identify CENP-C homologue in P. falciparum, protein sequences of the two conserved domains of human CENP-C, namely, the CENP-C signature motif and dimerization domain were used as queries for the BLAST analyses. The sequence comparisons revealed a putative CENP-C homologue [PF3D7_1021800] in the Plasmodium database [44]. The BLASTp between PfCENP-C, MIF2p and CENP-C shows the maximum sequence homology in the conserved regions of the CENP-C motif and the $\mathrm{C}$-terminal dimerization domain (Figure 1A,i) and thus these conserved domains were mapped in PfCENP-C (Figure 1A,ii). Percent pair-wise similarity of the fulllength PfCENP-C with full-length MIF2p and CENP-C were acquired by Needleman-Wunsch alignment. The full-length PfCENP-C shares 10.8 and $17.5 \%$ amino acid sequence similarities with MIF2p and CENP-C, respectively. The PfCENP-C-motif (amino acids 1105-1124) displays 30 and $63 \%$ amino acid sequence similarities with S. cerevisiae and human CENP-C motif, respectively (Figure 1B). The PfCENP-C dimerization domain (amino acids 1981-2074) shares 50 and $46 \%$ amino acid sequence similarities with S. cerevisiae and human dimerization domains, respectively (Figure $1 \mathrm{C}$ ). However, the amino terminal region of $P f C E N P-C$ exhibit low sequence similarity with the S. cerevisiae or human CENP-C. The in silico analysis thus suggested the presence of a putative CENP-C homologue in P. falciparum.

\section{PfCENP-C is constitutively expressed during all the intra- erythrocytic stages and localizes to the nuclear periphery}

To determine the presence and the expression pattern of $P f C E N P-C$ at the various intra-erythrocytic (IE) stages of the parasite, Western blot analysis was performed using anti-PfCENP-C antibodies on the parasite lysates prepared from the tightly synchronized ring, early trophozoite, late trophozoite and schizont stages (Figure 2A, lanes 1-4). The presence of PfCENP-C (244.9 kDa) is detected at all the stages but the expression is down regulated during the early trophozoite stage as compared to ring, late trophozoite and schizont stages, suggesting its differential constitutive expression at all the developmental stages of the IE cycle. The equal protein loading was confirmed by anti-GAPDH antibodies (Figure 2A, lanes 5-8). The sub-cellular localization of PfCENP-C at various IE stages by indirect immunofluorescence assays 
(A)

(B)
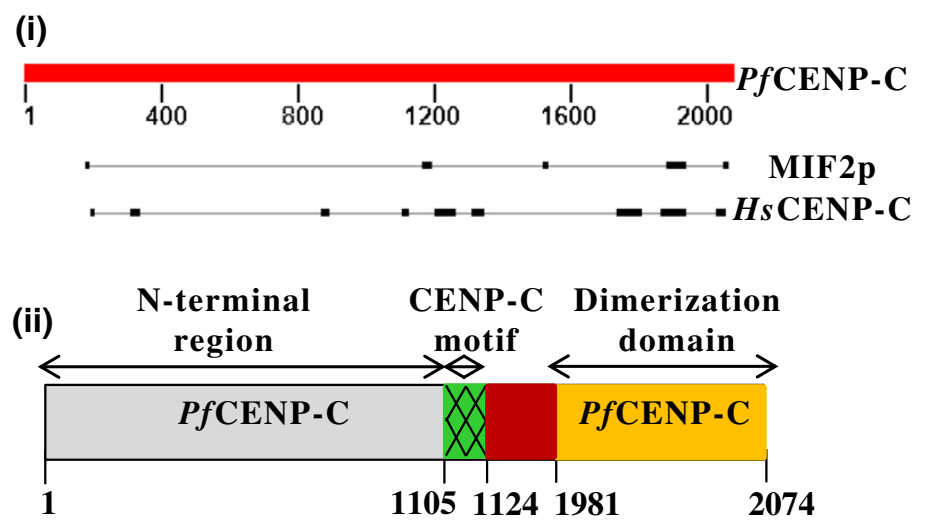

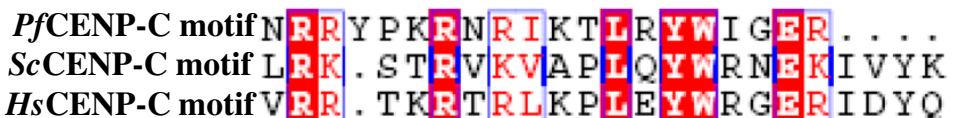

(C)

F1993 F1996

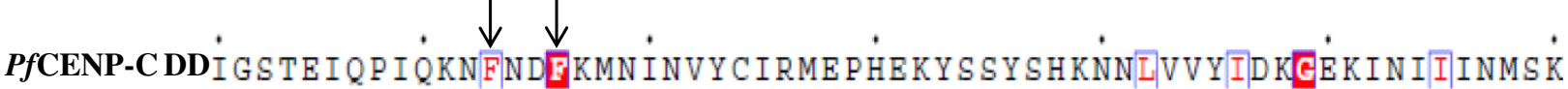
ScCENP-C DDFALEIMFDKHKEY|. .FASGILKL. PAIS. GQKKLSNSFRTY I T FHVI IEIVEVTV|CKNKF Hs CENP-C DD. . L KVY KT LDT P E. . EST T KL I L G PQEE KGKQHVG . . . QD I ¿V FYVN FED LLCTLHET PY

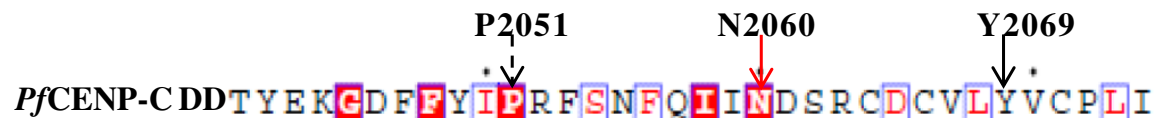

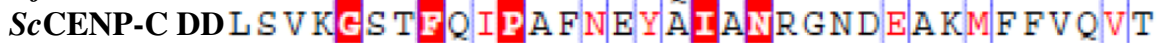
HsCENP-C DDI L S TED SEY VP S GNYYNIKNLRN EES V LL F T Q I|K

Figure 1 Identification of PfCENP-C and mapping of PfCENP-C conserved domains, the CENP-C motif and dimerization domain. (A) (i) The schematic representation of the regions of homology (black bars) between the full-length PfCENP-C, MIF2p (S. cerevisiae CENP-C) and human CENP-C using BLASTp analysis. The regions of maximum homology are confined to the centre and the C-terminus region. Percent pair-wise similarity of full-length PFCENP-C with full-length yeast MIF2p and human CENP-C were acquired by Needleman-Wunsch alignment. The full-length PfCENP-C shares 10.8 and $17.5 \%$ amino acid sequence similarities with MIF2p and CENP-C, respectively. (ii) The schematic representation of the PFCENP-C motif (green) and PFCENP-C dimerization domain (orange) based on the sequence comparisons and in silico analyses. The region before the PfCENP-C motif is represented as the N-terminal region (grey). The numbers indicate the amino acid positions. (B) Sequence comparison between the conserved CENP-C motifs. The CENP-C motif of $P$. falciparum shares 30 and $63 \%$ homology with SCCENP-C and HSCENP-C motifs, respectively. The red-shaded boxes represent the identical amino acids. (C) Sequence comparison of the dimerization domains (DD). The PFCENP-C-DD shows 50 and 46\% homology with the yeast and human CENP-C dimerization domains, respectively. The conserved amino acid residues in the dimerization domains of PFCENP-C and MIF2p are indicated by arrows. (Pf- Plasmodium falciparum; Sc- Saccharomyces cerevisiae; Hs- Homo sapiens).

using anti-PfCENP-C antibodies was performed. The immunofluorescence assays show that PfCENP-C localizes to the nuclear periphery at all the IE stages (Figure 2B). The PfCENP-C has also been found to colocalize with PfCENH3 at the nuclear periphery [39] which is consistent with the fact that CENP-C colocalizes with CENP-A throughout the cell cycle in other organisms like Drosophila and humans [20,52]. These results thus confirm the presence of endogenous PfCENP-C in the malaria parasite.
The motif and the dimerization domain of PfCENP-C functionally complements the MIF2p loss-of-function The CENP-C specifically recognizes CENP-A in the centromeric nucleosome and promotes the functional centromere-kinetochore assembly [7-9,12]. Mutations in the CENP-C motif and the dimerization domain lead to arrested growth, chromosome mis-segregation and mitotic delay $[6,25,34,36]$. In order to determine whether the putative PfCENP-C is a true homologue of CENP-C, the ability of the PfCENP-C domains, namely, the N-terminal region, 
(A)

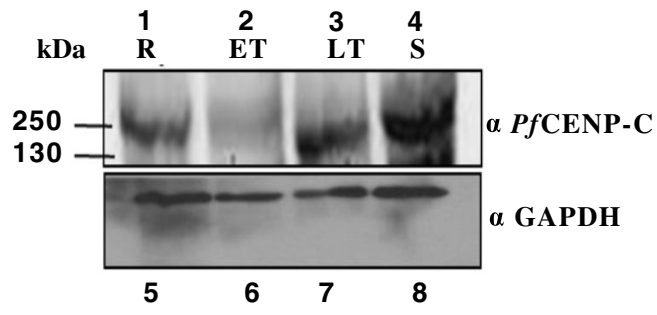

(B)

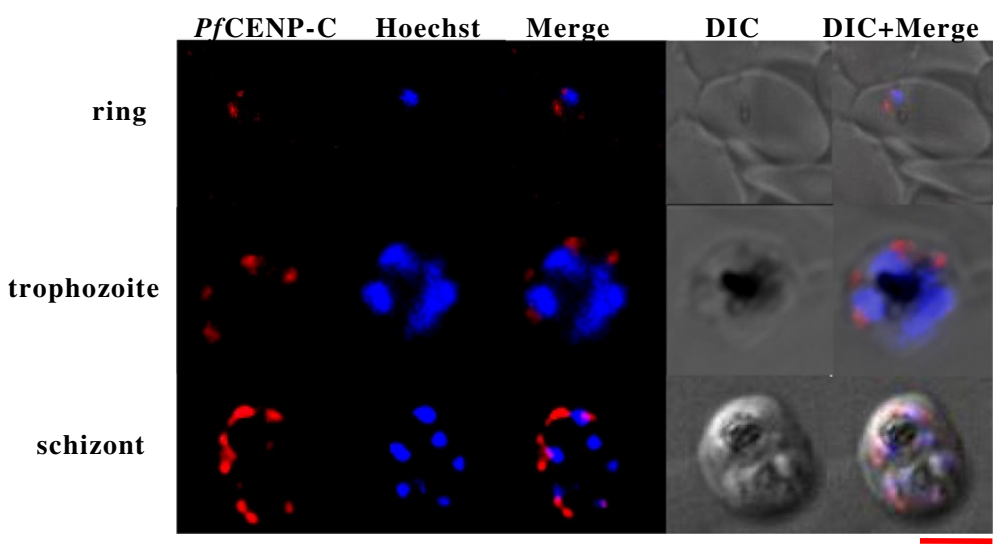

Scale bar $=5 \mu \mathrm{m}$

Figure 2 PfCENP-C exhibits constitutive differential expression throughout the intra-erythrocytic stages and localize to the nuclear periphery. (A) Stage-specific Western blot analyses of PFCENP-C on the whole cell parasite lysates from tightly synchronized parasites at ring (R), early trophozoite (ET), late trophozoite (LT) and schizont (S) stages were probed with anti-PfCENP-C (lanes 1-4) and anti-GAPDH (lanes 5-8) antibodies, respectively. (B) The confocal images for immunofluorescence assays representing the subcellular localization pattern of PfCENP-C (red) and the Hoechst stained nucleus (blue) at ring, trophozoite and schizont stages of $P$. falciparum, respectively. Scale bar $=5 \mu \mathrm{m}$.

PfCENP-C motif and PfCENP-C dimerization domain to functionally complement MIF2p depleted TS mutant strains, mif2-3 and mif2-2 [34,36] defective in mitotic chromosome segregation were tested.

Functional complementation assays with WT strain, mif2-3 and mif2-2 TS mutant yeast cells expressing PfCENP-C N-terminal region (NTD), PfCENP-C motif, PfCENP-C dimerization domain (DD), MIF2p, and an empty vector alone were performed at permissive $\left(23^{\circ} \mathrm{C}\right)$ and non-permissive $\left(37^{\circ} \mathrm{C}\right)$ temperatures (Figure $3 \mathrm{~A}$ and $3 \mathrm{~B}$, i-iii ; see Additional file $1 \mathrm{~A}$ and $\mathrm{B})$. At the permissive temperature, all the mif2-3 and mif2-2 TS mutants expressing the abovementioned constructs show normal growth (Figure 3A,i; see Additional file 1A). At the non-permissive temperature (Figure $3 \mathrm{~A}$,ii and iii), the untransformed mif2-3 and mif2-2 TS mutants show severely retarded growth. The mif2-3 TS mutants carrying the PfCENP-C motif and the PfCENP-C dimerization domain show partial and complete growth restoration, respectively, the mif2-2 TS mutants expressing PfCENP-
$\mathrm{C}$ motif and the PfCENP-C dimerization domain individually show only partial growth rescue. As compared to the complete growth rescue in the mif2-3 TS mutants, the PfCENP-C dimerization domain partially rescues the growth defects in the mif2-2 TS mutants. Both the TS mutants devoid of the $\mathrm{C}$-terminus region and expressing only the $\mathrm{N}$-terminal region of PfCENP-C show arrested growth at $37^{\circ} \mathrm{C}$ and fail to rescue the growth defects. The mif2-3 and mif2-2 TS mutants carrying the empty vector alone too, fail to grow at $37^{\circ} \mathrm{C}$. As expected, both the TS mutants expressing MIF2p show complete rescue in the growth at the non-permissive temperature. Ten-fold serial dilution assays (Figure 3B, i-iii; see Additional file 1B) for monitoring the viability in mif2-3 and mif2-2 TS mutant cells also corroborates the complementation ability of PfCENP-C motif and PfCENP-C dimerization domain in MIF2p-depleted cells, establishing that both motif and the dimerization domain of PfCENP-C are able to overcome the growth defects in its yeast counterpart but the PfCENP-C 


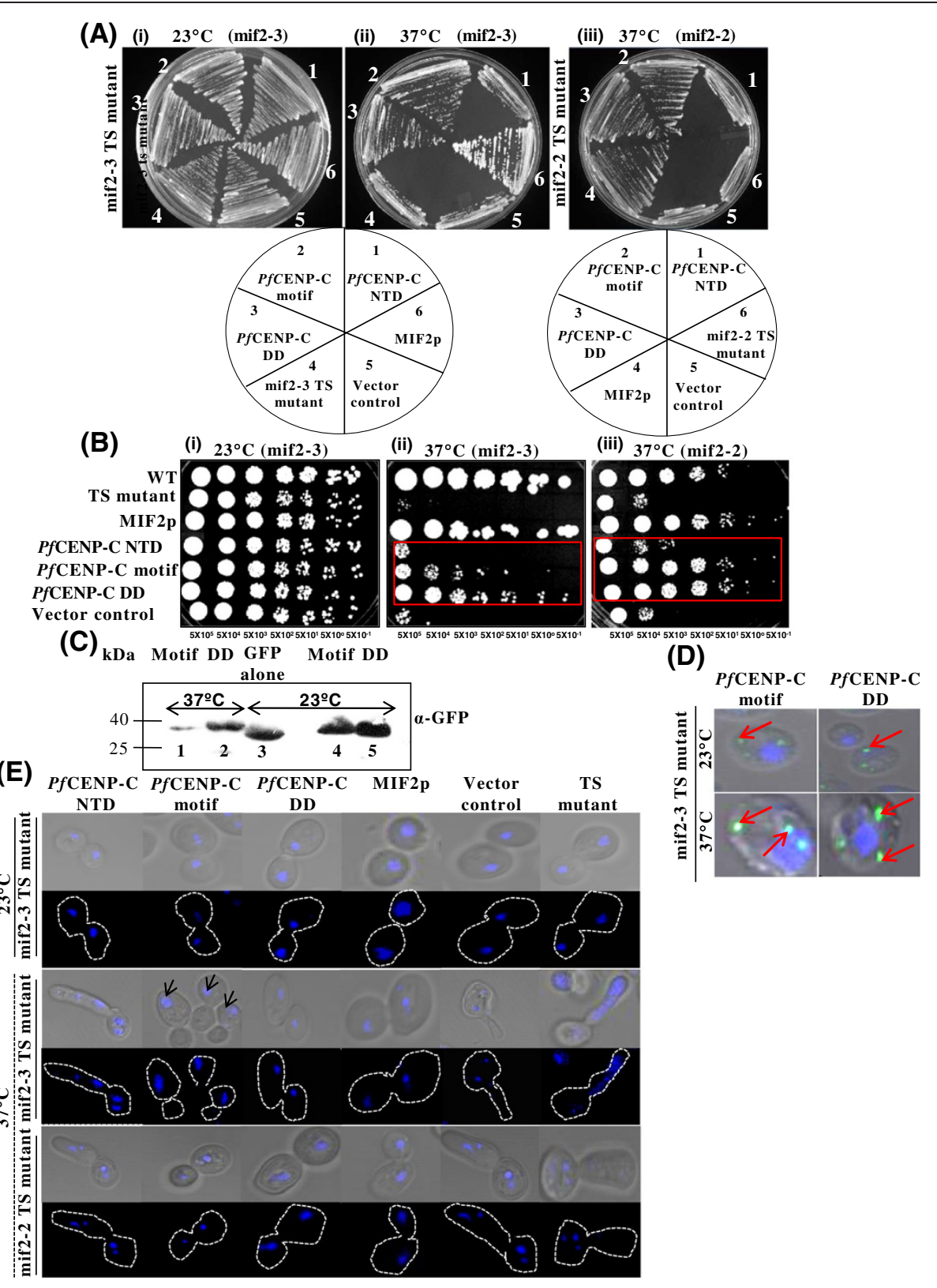

Figure 3 Functional complementation of Saccharomyces cerevisiae MIF2p by the PfCENP-C motif and PfCENP-C dimerization domain. (A and $\mathbf{B}$, i-iii) Growth and ten-fold serial dilution assays with mif2-3 (i and ii) and mif2-2 (iii) TS mutants expressing MIF2p, empty vector, various PFCENP-C domains: NTD, motif, and DD and the parent TS strains at 23 and $37^{\circ} \mathrm{C}$, respectively. (i) At $23^{\circ} \mathrm{C}$, the mif2-3 TS expressing abovementioned constructs display normal growth. (ii and iii) At $37^{\circ} \mathrm{C}$, TS mutants, expressing PFCENP-C-NTD, vector alone and the parent TS cells alone fail to grow, while MIF2p shows complete growth restoration. The mif2-3 TS mutants expressing PFCENP-C motif and DD show partial and complete growth rescue, respectively while in mif2-2 TS cells, both PFCENP-C motif as well as DD show partial growth rescue. The red boxes highlight the comparative growth of various PFCENP-C domains at $37^{\circ} \mathrm{C}$. (C) Western blot analysis showing the expression of GFP tagged PfCENP-C motif ( 29.68 kDa) and DD ( 38.16 $\mathrm{kDa}$ ) in mif2-3 TS mutants at 23 (lanes 4 and 5) and $37^{\circ} \mathrm{C}$ (lanes 1 and 2). (D) Confocal images of GFP tagged PfCENP-C motif and the DD (green) showing their mislocalization (red arrows) to the non-centromeric sites both at 23 and $37^{\circ} \mathrm{C}$. (E) Confocal images represent the phenotypes of mif2-3 and mif2-2 TS mutants carrying various constructs at 23 and $37^{\circ} \mathrm{C}$, respectively. At $23^{\circ} \mathrm{C}$, all the transformants show normal bud morphology with equal nuclear (blue) segregation. At $37^{\circ} \mathrm{C}$, like parent TS mutants, PFCENP-C-NTD and empty vector show aberrant bud morphology with fragmented nuclei. The PfCENP-C motif expressing mif2-3 and mif2-2 TS mutants shows chromosome mis-segregation (black arrows) and proper chromosome segregation, respectively. However, the PfCENP-C-DD expression in both the TS mutants restores the normal chromosome segregation. MIF2p expressing cells also exhibit normal phenotypes at $37^{\circ} \mathrm{C}$. N-terminal region-NTD, dimerization domain-DD. 
dimerization domain appears to exhibit better functional complementation ability as compared to the PfCENP-C motif.

To confirm the expression of the PfCENP-C motif and the PfCENP-C dimerization domain in the mif2-3 TS mutants, both the domains were tagged with GFP and Western blot analysis using anti-GFP antibodies were performed. The PfCENP-C motif and the PfCENP-C dimerization domain (DD) are expressed in the TS mutants at both the permissive as well as non-permissive temperatures (Figure 3C, lanes 1, 2, 4, and 5). The expression of CENP-C above the physiological level results in its mistargeting to the non-centromeric regions of the chromatin $[24,53]$. To check whether PfCENP-C motif and the PfCENP-C dimerization domain localize to the yeast centromeres, indirect immunofluorescence assays with anti-GFP antibodies were performed. Owing to the overexpression of PfCENP-C motif and the PfCENP-C dimerization domain, multiple discrete spots over the nuclei are observed indicating the mislocalization of both the domains (Figure 3D). The N-terminal of CENP-C has been shown to destabilize the excess CENP-C, which prevents the CENP-C accumulation to the non-centromeric sites and ensures its correct centromeric targeting [30]. Extrapolating these results for P. falciparum, the mislocalization of the signals in the mif2-3 TS mutants can be attributed to the absence of the $\mathrm{N}$-terminal region in the PfCENP-C motif and the PfCENP-C dimerization domain.

In $S$. cerevisiae, the deletion of the dimerization domain of MIF2p results in cells with TS phenotypes like abnormal large bud morphology, mitotic arrest and chromosome mis-segregation [6]. The large bud morphology is typically exhibited by cells arrested in S, G2 or $\mathrm{M}$ phases of the cell cycle and prolonged exposure to the non-permissive temperature leads to loss in viability [21]. To determine whether such phenotypic defects are rescued upon complementation of MIF2p by various PfCENP-C domains, the phenotypes of the mif2-3 and mif2-2 TS mutant cells deficient for MIF2p function as compared to the TS mutant cells expressing different PfCENP-C domains deletants, namely, the $\mathrm{N}$ - terminal region, CENP-C motif and the dimerization domain individually were examined and scored (Figures $3 \mathrm{E}$, see Additional file $1 \mathrm{C}, \mathrm{i}$-iii). At $23^{\circ} \mathrm{C}$, normal bud morphology and proper chromosome segregation is observed in both the TS mutants carrying the different PfCENP-C deletion mutants. At $37^{\circ} \mathrm{C}$, the majority of the parent TS mutant cells (mif2-3 and mif2-2) and the vector control show aberrant large bud morphology with dispersed and/or fragmented nuclei along with unviable cells. Similarly, at $37^{\circ} \mathrm{C}$, both the TS mutants expressing the $\mathrm{N}$-terminal region (NTD) of PfCENP-C show predominantly large bud morphology with fragmented nuclear mass along the mother-bud neck and a subset of unviable cells lacking detectable nuclear staining. These abnormal phenotypes reflect impaired chromosome segregation on MIF2p lossof-function. At $37^{\circ} \mathrm{C}$, the mif2-3 TS mutants expressing the PfCENP-C motif show normal bud morphology but with chromosome mis-segregation (Figure 3E, black arrows) as cells show a single unsegregated G2 nucleus in the mother cell while the mif2-2 TS mutants expressing the PfCENP-C motif show normal bud morphology and proper chromosome segregation. The majority of mif2-3 and mif2-2 TS mutant cells expressing PfCENP-C dimerization domain (DD) show normal bud morphology with proper chromosome segregation apparent by the intracellular location of the nucleus that is equally segregated between the bud and the mother cell. The TS mutants expressing budding yeast MIF2p also show restoration of the normal phenotype at $37^{\circ} \mathrm{C}$. The ability of PfCENP-C motif in mif2-2 TS mutants and the PfCENP-C dimerization domain in mif2-2 and mif2-3 TS mutants to rescue the mitotically arrested cells and overcome the chromosome segregation defects demonstrates their role in mediating accurate chromosome segregation. Collectively, these results suggest that the putative PfCENP-C is a true CENP$\mathrm{C}$ homologue.

\section{The dimerization domain of PfCENP-C harbours functionally important F1993, F1996 and Y2069 residues}

The dimerization domain of CENP-C is essential for centromere function, proper structuring of centromeric chromatin and in vivo kinetochore assembly [4,6,27,29,34]. To decipher the underlying residues essential for the functions of PfCENP-C dimerization domain, point mutations in some of the conserved amino acid residues of this domain were incorporated. In budding yeast MIF2p, the residues Y451, F452 and F523 within the dimerization domain are shown to be essential for its dimerization ability upon which mutation results in arrested growth while the mutation of another conserved residue T488 which is present outside the dimer interface is dispensable for growth [6]. The multiple sequence alignment of the dimerization domain between $P$. falciparum and the budding yeast shows that the amino acid residues F1993, F1996 and Y2069 of PfCENP-C dimerization domain corresponds to the residues Y451, F452 and F523 of MIF2p dimerization domain, respectively (Figure $1 \mathrm{C}$, solid black arrows). As the PfCENP-C dimerization domain contains an 'isoleucine' instead of 'T488' at the analogous position in MIF2p, another conserved residue, N2060 (Figure 1C, solid red arrow) in PfCENP-C corresponding to N514 in MIF2p dimerization domain was mutated to ' $\mathrm{A}$ ' to determine its role on growth and chromosome segregation. Since the functional dimerization domain is required for the normal growth in yeast [6], the functional complementation assays were performed to investigate the effect of PfCENP-C dimerization domain mutants PfCENP-C ${ }^{\mathrm{F} 1993 \mathrm{~A}}$, PfCENP-C $\mathrm{C}^{\mathrm{F} 1996 \mathrm{~A} \text {, }}$ 
PfCENP-C ${ }^{\mathrm{Y} 2069 \mathrm{~A}}$ and PfCENP-C $\mathrm{C}^{\mathrm{N} 2060 \mathrm{~A}}$ on the growth of TS mif2-3 and mif2-2 cells at the permissive (Figure 4A and $\mathrm{B}$, i; see Additional file $2 \mathrm{~A}$ and $\mathrm{B}$ ) and non-permissive (Figure 4A and B, ii-iii) temperatures. The complementation (Figure 4A, i-iii; see Additional file 2A) and ten-fold serial dilution (Figure 4B, i-iii; see Additional file 2B) assays for monitoring the viability and phenotypes of mif2-3 and mif2-2 TS mutant cells, expressing the various PfCENP-C dimerization domain mutants individually were tested. At the permissive temperature (Figure 4A and B, i; see Additional file 2Aand B), the mif2-3 and mif2-2 TS cells expressing different PfCENP-C dimerization domain show normal growth rate and colony size as the TS mutants expressing the WT PfCENP-C dimerization domain (DD). At the non-permissive temperature (Figure $4 \mathrm{~A}$ and $\mathrm{B}$,ii and iii), unlike the WT PfCENP-C dimerization domain, the mif2-3 and mif2-2 TS mutant cells expressing PfCENP-C ${ }^{\mathrm{F} 1993 \mathrm{~A}}$, PfCENP-C $\mathrm{C}^{\mathrm{F} 1996 \mathrm{~A}}$ and PfCENP-C $\mathrm{C}^{\mathrm{Y} 2069 \mathrm{~A}}$ dimerization domains show significant growth reduction while both the TS mutants expressing PfCENP-C ${ }^{\mathrm{N} 2060 A}$ show unimpaired growth. The growth assays demonstrate the essentiality of the residues F1993, F1996 and Y2069 within the dimerization domain of PfCENP-C while the residue $\mathrm{N} 2060$ is not essential for PfCENP-C functions.

Further, the phenotypic changes exhibited by the dimerization domain mutants PfCENP-C ${ }^{\text {F1993A }}$, PfCENP$\mathrm{C}^{\mathrm{F} 1996 \mathrm{~A}}$, PfCENP-C $\mathrm{Y}^{\mathrm{Y} 2069 \mathrm{~A}}$ and PfCENP-C $\mathrm{C}^{\mathrm{N} 2060 \mathrm{~A}}$ were assessed at 23 and $37^{\circ} \mathrm{C}$, respectively (Figure $4 \mathrm{C}$, see Additional file $2 \mathrm{C}$ ). At $23^{\circ} \mathrm{C}$, mif2-3 and mif2-2 TS mutant cells expressing different PfCENP-C dimerization domains show proper chromosome segregation and normal bud morphology. At $37^{\circ} \mathrm{C}, \sim 90-94 \%$ of mif2-3 and mif2-2 TS mutant cells expressing PfCENP-C ${ }^{\text {F1993A }}$, PfCENP-C ${ }^{\mathrm{F} 1996 \mathrm{~A}}$ and PfCENP-C ${ }^{\mathrm{Y} 2069 \mathrm{~A}}$ are mitotically arrested and unviable. This is evident by the aberrant bud morphologies with fragmented nucleus or loss of nuclear staining. These abnormal phenotypes are attributable to the failure of PfCENP-C dimerization domain mutants to dimerize resulting in impaired growth, defective chromosome segregation and loss of viability. Both the TS mutants expressing PfCENP-C ${ }^{\mathrm{N} 2060 \mathrm{~A}}$ show normal bud morphology accompanied with proper chromosome segregation. The data presented here identify some of the key residues that are essential for the formation of a functional dimerization domain. This further demonstrates the role of the PfCENP-C dimerization domain in mediating the in vivo CENP-C functions.

In $S$. cerevisiae, among the various mif $2 \mathrm{p}$ TS mutants (chromosome segregation mutants), the mif2-3 TS mutant is very well characterized. The mif2-3 TS mutant has a point mutation, $\mathrm{P} 505 \mathrm{~L}$ in the MIF2p dimerization domain where proline at position 505 is substituted by leucine $[34,36]$. At the non-permissive temperature, the MIF2p ${ }^{\text {P505L }}$ mutation confers the aberrant TS phenotypes to the mif2-3
TS mutants and renders the dimerization domain nonfunctional $[6,34,36]$. The amino acid residue proline is conserved in MIF2p, CENP-C and also in P. falciparum CENP-C at the analogous position (Figure $1 \mathrm{C}$, broken black arrow). To confirm the complementation ability of PfCENP-C dimerization domain, the conserved proline in the PfCENP-C dimerization domain was substituted with leucine and the mutant PfCENP-C $\mathrm{C}^{\mathrm{P} 2051 \mathrm{~L}}$ corresponding to the MIF2 $\mathrm{p}^{\mathrm{P} 505 \mathrm{~L}}$ mutation in the TS mif2-3 mutant was used for the studies. Figures $4 \mathrm{D}$ and $\mathrm{E}$ show the functional complementation and ten-fold serial dilution assays of mif2-3 TS mutants expressing PfCENP-C $\mathrm{C}^{\mathrm{P} 2051 \mathrm{~L}}$ dimerization domain as compared to the mif2-3 TS mutants expressing the WT PfCENP-C dimerization domain at permissive and non-permissive temperatures, respectively. At $23^{\circ} \mathrm{C}$, TS mutants expressing the PfCENP-C $\mathrm{C}^{\mathrm{P} 2051 \mathrm{~L}}$ dimerization domain display normal growth like the WT PfCENP-C dimerization domain. However at $37^{\circ} \mathrm{C}$, similar to the mif2-3 TS mutants and vector control, the TS mutants carrying the PfCENP-C $\mathrm{C}^{\mathrm{P} 2051 \mathrm{~L}}$ dimerization domain too show complete growth arrest while normal growth is observed in the mif2-3 TS mutants expressing WT nonmutated PfCENP-C dimerization domain. These results indicate that the PfCENP-C $\mathrm{C}^{\mathrm{P} 2051 \mathrm{~L}}$ confers the TS phenotype.

The analysis was extended by examining the phenotypes exhibited by mif2-3 TS mutants expressing PfCENP$\mathrm{C}^{\mathrm{P} 2051 \mathrm{~L}}$ dimerization domain both at permissive and nonpermissive temperatures (Figure $4 \mathrm{~F}$ ). At $23^{\circ} \mathrm{C}$, majority of mif2-3 TS mutants expressing PfCENP-C $\mathrm{C}^{\mathrm{P} 2051 \mathrm{~L}}$ dimerization domain show normal bud morphology accompanied with chromosome mis-segregation phenotypes. Whereas at $37^{\circ} \mathrm{C}$, similar to the untransformed mif2-3 TS mutant cells, the TS mutants expressing PfCENP-C ${ }^{\mathrm{P} 2051 \mathrm{~L}}$ dimerization domain show aberrant bud morphology, G2/M-arrest, elevated chromosome mis-segregation phenotypes, including unviable cells. The chromosome mis-segregation phenotype of TS mutants carrying the PfCENP-C ${ }^{\text {P2051L }}$ dimerization domain even at the permissive temperature, (Figure 4F, black arrow) indicates that despite normal growth, the MIF2p functions are compromised. These chromosome segregation defects are further exacerbated at the non-permissive temperature, reflecting the loss of MIF2p function in the absence of a functional dimerization domain. The inability of the PfCENP-C $\mathrm{C}^{\mathrm{P} 2051 \mathrm{~L}}$ dimerization domain to complement MIF2p can be attributed to the absence of a functional dimerization domain, which in turn fails to overcome the chromosome segregation defects in the mif2-3 TS mutants.

\section{PfCENP-C dimerization domain is required for maintaining the mitotic spindle integrity}

In $S$. cerevisiae, MIF2p is required for the maintenance of normal spindle structures. Fluorescence and electron microscopy in the large budded TS mif2-3 and mif2-2 


\section{(A)}
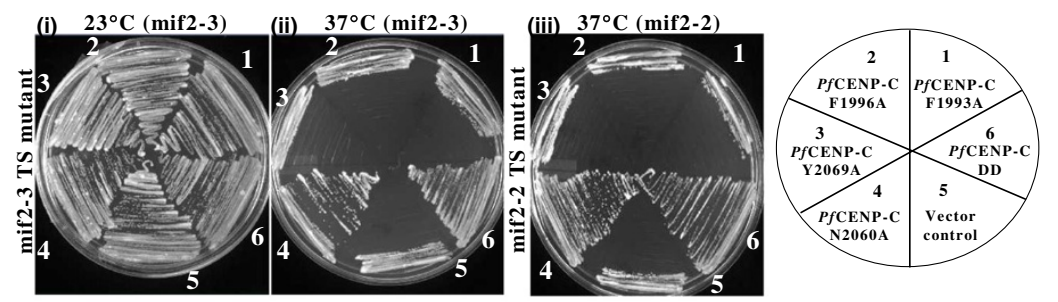

(B)

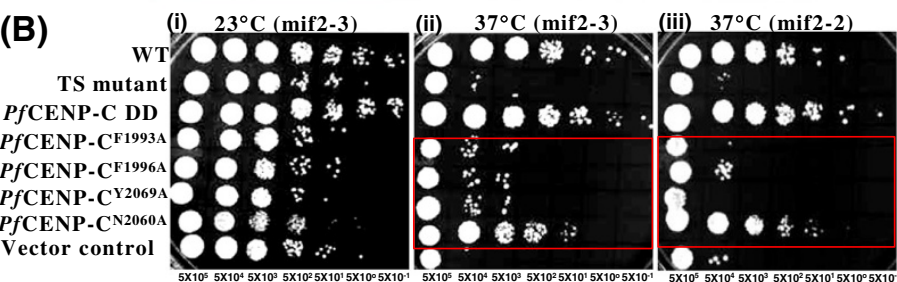

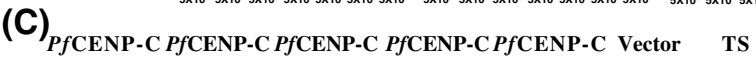
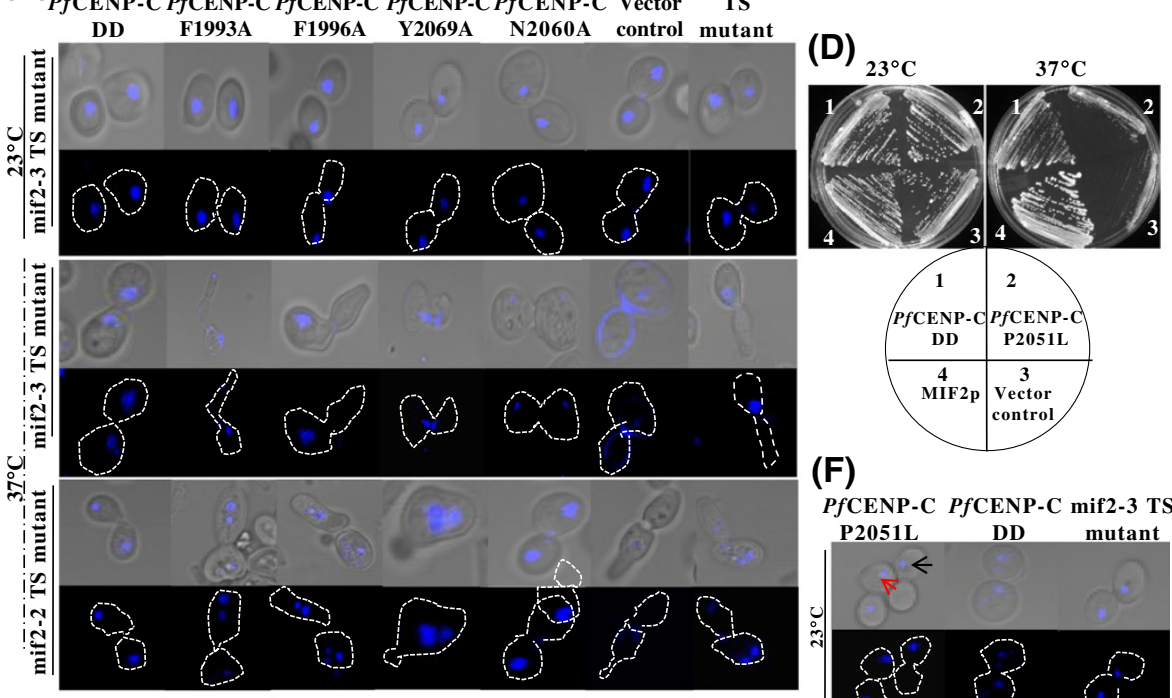

(F)

PfCENP-C PfCENP-C mif2-3 TS

(E)
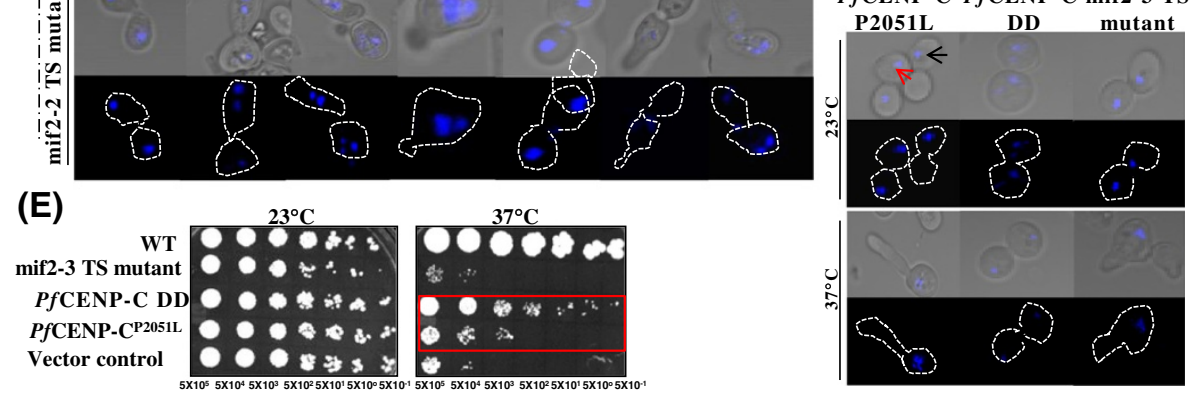

Figure 4 Functionally essential residues lie within the PfCENP-C dimerization domain. (A and $\mathbf{B}$, i-iii) Complementation and ten-fold serial dilution assays with the PFCENP-C dimerization domain (DD) carrying mutations at the conserved residues essential for the CENP-C dimerization. (i) At $23^{\circ} \mathrm{C}$, the different PfCENP-C-DD mutants, PfCENP-C C1993A, PfCENP-C $\mathrm{C}^{11996 \mathrm{~A}}$, PfCENP-C ${ }^{\mathrm{Y} 2069 \mathrm{~A}}$, PfCENP-C $C^{\mathrm{N2} 2060 \mathrm{~A}}$ and vector control show normal

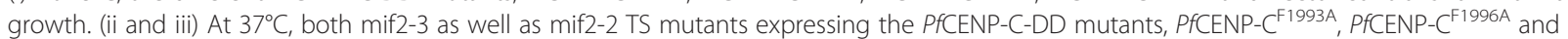
PFCENP-C ${ }^{\mathrm{Y} 2069 \mathrm{~A}}$ show complete growth arrest while the WT PFCENP-C-DD and PfCENP-C ${ }^{\text {N2060A }}$ show growth rescue. The red boxes show the comparative growth patterns of WT PfCENP-C-DD and various PfCENP-C-DD mutants in mif2-3 and mif2-2 TS mutants at $37^{\circ} \mathrm{C}$. (C) Confocal images of

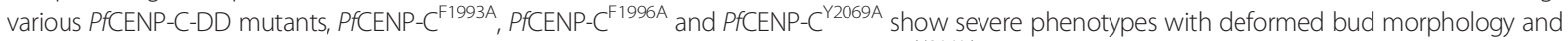
dispersed and /or fragmented nuclear staining in the TS mutants while the PfCENP-C ${ }^{\mathrm{N} 2060 \mathrm{~A}}$ show normal phenotype at $37^{\circ} \mathrm{C}$. (D and E) The functional complementation assays with PFCENP-C $C^{\text {2051L }}$ corresponding to mif2-3 TS MIF2 $p^{\text {P505L }}$ mutation shows growth arrest at $37^{\circ} \mathrm{C}$ while the non-mutated WT PFCENP-C-DD facilitates growth at $37^{\circ} \mathrm{C}$ in mif2-3 TS mutant cells (highlighted with red box). (F) The confocal images representing the gain-or loss-of-

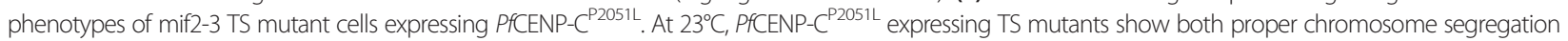
(red arrows) as well as chromosome mis-segregation phenotype (black arrow) evident by the nucleus (blue) localization. At $37^{\circ} \mathrm{C}$, PfCENP-C ${ }^{2} 2051 \mathrm{~L}$ expressing TS mutants show abnormal phenotypes and most of the mif2-3 cells expressing PFCENP-C ${ }^{\text {P2051L }}$ are unviable. The WT non-mutated PfCENP-C-DD displays normal chromosome segregation phenotypes at both 23 and $37^{\circ} \mathrm{C}$, respectively.

cells have revealed the presence of short, discontinuous and broken spindles [6,21]. To determine the spindle morphology in mif2-3 and mif2-2 TS mutant cells expressing various PfCENP-C domains, indirect immunofluorescence assays by staining the transformed mif2-3 and mif2-2 TS strains with anti-tubulin antibodies 
were performed. The confocal images (Figure 5A, see Additional file 3A) show the mitotic spindle morphologies of both the TS mutants alone and the TS mutants expressing PfCENP-C N-terminal region, PfCENP-C motif, PfCENP-C dimerization domain, and MIF2p individually at 23 and $37^{\circ} \mathrm{C}$, respectively. At permissive temperature, continuous spindles with properly segregated nuclear masses across the budding cells are observed in the TS mutants expressing different PfCENP-C domains. At $37^{\circ} \mathrm{C}$, both the TS mutants expressing the PfCENP-C $\mathrm{N}$-terminal region and vector alone show discontinuous, unelongated and broken spindles as these cells with abnormal morphologies show no continuous tubulin staining along the nuclear mass, which is in concordance with the previous study of Brown et al. [21]. However, the mif2-3 and mif2-2 TS mutants expressing only the PfCENP-C dimerization domain could rescue the abnormal spindle morphologies as continuous spindles connecting the two discrete nuclear masses in the budding cells are seen in $\sim 65 \%$ and $\sim 40 \%$ of the rescued cells, respectively. The mif2-3 and mif2-2 TS mutants expressing the PfCENP-C motif show $\sim 82 \%$ and $\sim 68 \%$ cells with broken spindles, respectively. At $37^{\circ} \mathrm{C}$, normal spindle morphologies are also observed in the TS mutants expressing yeast MIF2p. The aberrant spindle morphologies in the TS cells are apparent as compared to the TS cells rescued by PfCENP-C dimerization domain. The presence of discontinuous spindles could be due either to the loss in connection between the spindles or an abrupt breaking of the spindles [21]. The discontinuous spindles might hamper the pole-ward movement of sister chromatids during anaphase ultimately contributing to the defective chromosome segregation [21]. The restoration of normal mitotic spindle structures on complementation with the dimerization domain of PfCENP-C also demonstrates the PfCENP-C dimerization domain-mediated proper chromosome segregation in the MIF2p deficient cells. The ability of dimerization domain to rescue the spindle defects in TS mutant cells raised the question whether the mitotic spindle integrity is disrupted on expression of PfCENP$\mathrm{C}$ dimerization domain mutants. The confocal images (Figure 5B, see Additional file $3 \mathrm{~B}$ ) show that at non-permissive temperature, none of the PfCENP-C dimerization domain mutants (PfCENP-C ${ }^{\text {F1993A }}$, PfCENP$\mathrm{C}^{\mathrm{F} 1996 \mathrm{~A}}$ and PfCENP-C $\mathrm{C}^{\mathrm{Y} 2069 \mathrm{~A}}$ ) could rescue the abnormal spindle morphology which is evident by the presence of disintegrated spindles in the mif2-3 and mif2-2 TS cells expressing all the above mentioned PfCENP-C dimerization domain mutants. The mif2-3 and mif2-2 TS mutants expressing PfCENP-C ${ }^{\mathrm{N} 2060 \mathrm{~A}}$ show intact normal spindles confirming its dispensable role in chromosome segregation. The loss of spindle integrity in the absence of dimerization domain is consistent with a defective centromere protein. These results confirm the essential role of the PfCENP-C dimerization domain in maintaining the mitotic spindles integrity during chromosome segregation.

\section{PfCENP-C is enriched at Plasmodium centromeres and interacts with PfCENH3 and a-tubulin in vivo}

To determine the enrichment of PfCENP-C on centromeric regions in vivo, ChIP assays were performed as previously described [49,50] with PfCENP-C and PfCENH3 antibodies (Figure 6A). As the centromeres are occupied by PfCENH3 on all the P. falciparum centromeres [40], ChIP with anti-PfCENH3 was used as a positive control. Semiquantitative PCR analysis on PfCENP-C and PfCENH3 immunoprecipitated samples using the set of primers specific for the centromeric regions of six $P$. falciparum chromosomes (CEN 1, 2, 3, 4, 9, and 12) [40] were carried out. To validate the specificity of the assay, control primers from regions lying upstream $(+\mathrm{CEN})$ and downstream (-CEN) of each centromere tested were designed (see Table 1 for the control primers) for the semi-quantitative PCR analyses on the ChIP samples followed by the quantification of the gel band intensities. The ChIP-PCR analyses show that like PfCENH3, PfCENP-C is also enriched on all the six $P$. falciparum centromeric regions analysed. However, at the non-centromeric sites, upstream as well as downstream of each centromere, very weak or no enrichment of PfCENH3 and PfCENP-C is observed. The in vivo enrichment of PfCENP-C on the centromeric regions confirms its functions as a centromere protein in $P$. falciparum. Like PfCENH3, the results also establish PfCENP-C as the centromere binding protein in the parasite.

The co-occupancy of PfCENH3 and PfCENP-C on the centromeres and their co-localization throughout the cell cycle [39] prompted to check the in vivo interactions of PfCENH3with PfCENP-C by co-immunoprecipitation (co-IP) assay. The pull-down of PfCENH3 using antiPfCENP-C antibodies and vice-versa were performed. The presence of PfCENH3 and PfCENP-C in the pulldown immunocomplexes was confirmed by the Western blot analyses (Figure 6B, panels i-vi). The co-IP assays with anti-PfCENP-C show its association with PfCENH3 and $\alpha$-tubulin, marker for mitotic spindles (Figure 6B, panels ii and iii). Similarly, co-IP assays using antiPfCENH3 could pull down both PfCENP-C and $\alpha$-tubulin (Figure 6B, panels v and vi). The association of PfCENP-C with PfCENH3 and mitotic spindles suggests the conserved mechanism of centromere-kinetochore assembly in P. falciparum.

\section{Discussion}

PfCENP-C is constitutively expressed throughout the Plasmodium cell cycle and like PfCENH3, localizes to the centromeres at the perinuclear regions. Employing functional complementation of MIF2p by PfCENP-C domains, this study reports the functions of PfCENP-C, emphatically 


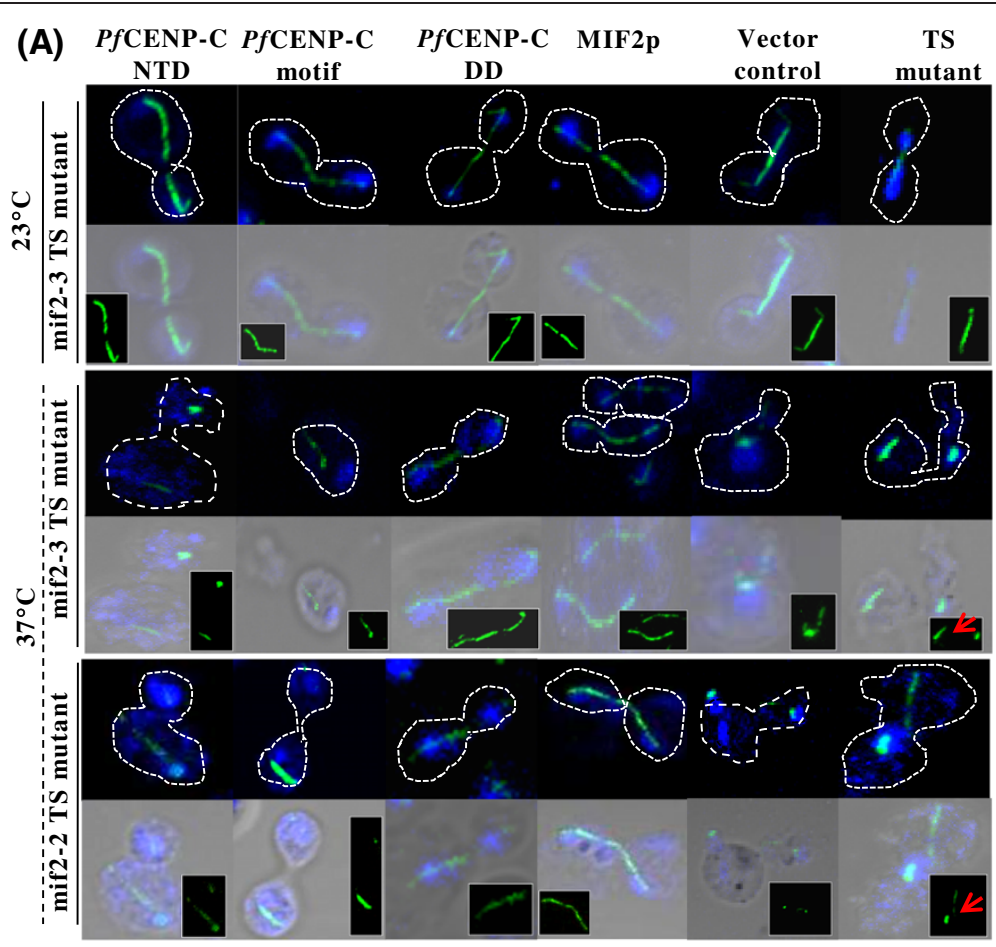

(B) PfCENP-C PfCENP-C PfCENP-C PfCENP-C
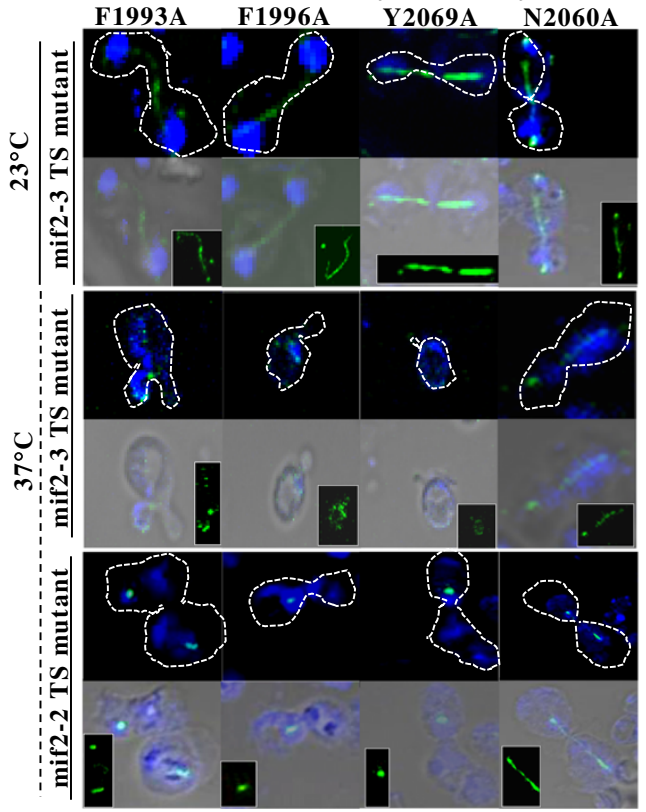

Figure 5 The mitotic spindle integrity is maintained by the PfCENP-C dimerization domain. (A) Confocal images showing the mitotic spindle (green) and the Hoechst stained nucleus (blue) of the mif2-3 and mif2-2 TS mutant cells expressing the PfCENP-C-NTD, -motif and -DD, region, MIF2p and empty vector alone at 23 and $37^{\circ} \mathrm{C}$, respectively. At $23^{\circ} \mathrm{C}$, all the mif2-3 TS mutants expressing different constructs show normal spindle structure connecting the nuclear masses across the bud and mother cell. At $37^{\circ} \mathrm{C}$, both in mif2-3 and mif2-2 TS mutants except the PFCENP-C-DD and MIF2p, all the other constructs show abnormal and discontinuous spindles. The spindle morphology (green) is also shown in the insets. The broken spindle in the mif2-3 and mif2-2 TS mutant cells are shown by red arrows in the insets. (B) The confocal images representing the disintegrated mitotic spindle morphologies in mif2-3 and mif2-2 TS mutant cells expressing the PFCENP-C-DD mutants, PFCENP-C ${ }^{\text {F1993A }, ~ P F C E N P-C ~}{ }^{\text {F1996A }}$ and PFCENP-C ${ }^{22069 A}$ while PFCENP-C ${ }^{\text {N2060A }}$ show normal mitotic spindles at $37^{\circ} \mathrm{C}$. NTD- N-terminal region; DD-dimerization domain. 

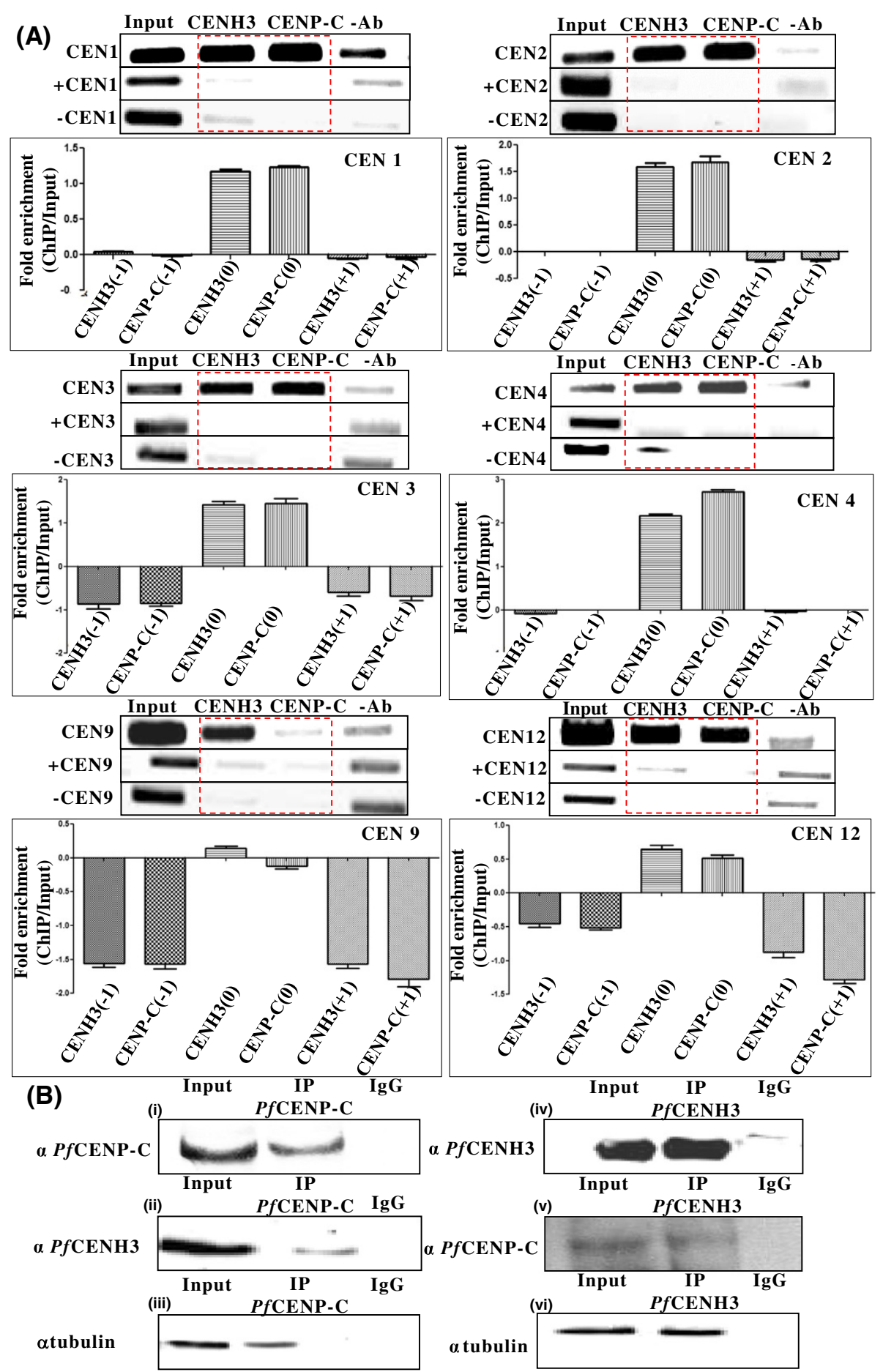

Figure 6 In vivo enrichment of PfCENP-C on the Plasmodium centromeres and its in vivo interaction with PfCENH3 and mitotic spindles. (A) ChIP-PCR assay showing the enrichment of PFCENP-C on the six P. falciparum centromeres (CEN 1, 2, 3, 4, 9, and 12) as Compared to the non centromeric regions (red dotted boxes). +CEN and -CEN represents the non-centromeric regions lying upstream and downstream of each $P$. falciparum centromere, respectively. The non-centromeric regions show weak or no recruitment of PfCENH3 and PfCENP-C for all the six P. falciparum centromeres tested. The fold enrichment (ChIP over input) was determined for each centromere in comparison with the non-centromeric regions by quantifying the gel band intensities using Alphalmager. In the graphs ( $x$-axis), CENH3 (+1), CENP-C (+1) and CENH3 (-1), CENP-C (-1) represents the ChIP with PFCENH3 and PFCENP-C at regions upstream and downstream to the centromeres, respectively. CENH3 (0) and CENP-C (0) in the $x$-axis of the graphs represent the PFCENH3 and PFCENP-C enriched centromeric regions, respectively. The $y$-axis represents the fold enrichment (ChIP over input) for each chromosome tested. (B) Immunoprecipitation assays with PfCENP-C (panels, i-iii) and PfCENH3 (panels, iv-vi) show the interaction of PFCENP-C with PFCENH3 (panel ii) and mitotic spindles (panel iii). PFCENH3 also interacts with PFCENP-C (panel v) and tubulin (panel vi). 
its dimerization domain to understand its role in chromosome segregation and mitotic events. The results establish PfCENP-C as a true homologue of CENP-C. Thus, this study delineates the functions of the dimerization domain of PfCENP-C in P. falciparum.

In yeast and humans, the dimerization domain of CENP$\mathrm{C}$ is reported to be essential for growth, self association and kinetochore assembly. The ability of PfCENP-C motif and the dimerization domain to rescue the loss of function in the TS MIF2p mutant cells is evident by the restoration of normal phenotype and proper chromosome segregation. The results indicate that the PfCENP-C dimerization domain is more efficient than the PfCENP-C motif in overcoming the MIF2p loss-of-function. The inability of the Cterminal deletion mutants of PfCENP-C to overcome the growth and chromosome segregation defects indicate that the N-terminal region of PfCENP-C is dispensable for the growth but the possibility of this region having other functions cannot be ruled out and needs experimental

\section{Table 1 Set of primers for amplification of $\boldsymbol{P}$. falciparum} non-centromeric regions

\begin{tabular}{|c|c|}
\hline Primer name & Nucleotide sequence $\left(5^{\prime}\right.$ to $\left.3^{\prime}\right)$ \\
\hline NONCEN 1-FP & CATCACCTGTCATAGCAACTG \\
\hline NONCEN 1-RP & GTTGTATAATGGAAGAGAATTTGAAG \\
\hline NONCEN 1-FP & GTAAAAGGAAAGAAGAAAAGAGTAGAG \\
\hline NONCEN 1-RP & ACAATCATTAGTAACATATTCACGAAC \\
\hline NON-CEN 2-FP & GATAAAAATGTTATATTAGGTTCAAAAG \\
\hline NON-CEN 2-RP & GGAATATGGTATCTCTATCTATAG \\
\hline NON-CEN 2-FP & ACCCAAGTTAGTTAAGTTAAGACC \\
\hline NON-CEN 2-RP & TTCACTAACATAGGTCTTACATTC \\
\hline NON-CEN 3-FP & ACCTATGTTAGTATCTACATGACC \\
\hline NON-CEN 3-RP & AGGTCTTACTCTCACTGATATAG \\
\hline NONCEN 3-FP & TACATCATACCCACCGTCCAATC \\
\hline NONCEN 3-RP & GATTTCAAGTCATTATITGTITGTACGAG \\
\hline NONCEN 4-FP & ATTATTATTCCCCTTTCATCATTITATG \\
\hline NONCEN 4-RP & ATAGCGGAAGTCCTAGAACATC \\
\hline NONCEN 4-FP & AAAATATGTAGAGGACTATGAATCAGATG \\
\hline NONCEN 4-RP & AATAAATGATTATCCTCTACATCTTGATTC \\
\hline NONCEN 9-FP & GCAAATACTAAATGTGTTCGTATGGG \\
\hline NONCEN 9-RP & TATTTGGATCAATGTACCGAATTTACTC \\
\hline NONCEN 9-FP & TATATTCTAAGAAAAATCTGAAATCCTTC \\
\hline NONCEN 9-RP & GACTAAGGATATGTGGAATCGTG \\
\hline NONCEN 12-FP & GACATTCAGGGGATCATTATTACATTC \\
\hline NONCEN 12-RP & TGATTGCTTTACTATAAATAATAAGAATGAAG \\
\hline NONCEN 12-FP & CTTCAAATAATTTTATATCTACATCCGTITTG \\
\hline NONCEN12-RP & GAAAAATGTGATGAGAAAGTAGGAAGTC \\
\hline
\end{tabular}

The set of primers used for the amplification of non-centromeric regions upstream and downstream of the $P$. falciparum centromeres. NONCEN refers to non-centromeric regions and the numbers represent the chromosome numbers. validation. The possibility of interaction of endogenous N-terminal region of MIF2p with PfCENP-C motif and dimerization domain resulting in a fully functional protein that mediates the proper chromosome segregation also cannot be ruled out.

CENP-C when expressed at physiological level localizes exclusively to the centromeres but on overexpression, displays mistargeting to the non-centromeric chromatin regions [53]. The overexpressed CENP-C in chicken DT40 cells localizes to both the centromere as well as the chromosomal arms indicating its mislocalization [24]. The mislocalization of PfCENP-C motif and PfCENP-C dimerization domain in the transformants might be the consequence of its overexpression under the strong $\mathrm{ADH}$ promoter in the p415ADH vector, which prevents the specific centromeric localization of these domains. Alternatively, as the $\mathrm{N}$-terminal domain of CENP-C regulates the CENP-C expression by destabilizing the excess CENP-C, an inherent mechanism to ensure centromere-specific localization of CENP-C [30] and as both the PfCENP-C motif and PfCENP-C dimerization domain constructs lack the N-terminal region of PfCENP-C, suggests the possibility that the mislocalization of the PfCENP-C domains might be due to the absence of destabilizing function performed by its N-terminal region.

In budding yeast, the mutations in the conserved residues Y451S, F452A and F523A within the MIF2p dimerization domain have been shown to break the contacts between the dimer, possibly by disrupting the conformation of strand connecting the two $\beta$-sheets of MIF2p, which destabilizes the dimerization domain [6]. The studies presented here indicate that the corresponding PfCENP-C residues F1993, F1996 and Y2069, respectively, too are essential for the stable and functional dimerization domain unlike the conserved PfCENP-C N2060 residue, which is dispensable. The TS mutant mif2-3 harbours a MIF2p ${ }^{\text {P505L }}$ mutation in its dimerization domain that is responsible for the TS characteristics and the aberrant phenotypes exhibited by the mif2-3 TS mutants at the non-permissive temperature $[6,34,36]$. The inability of the PfCENP-C ${ }^{\text {P2051L }}$ dimerization domain mutant corresponding to MIF2 $\mathrm{p}^{\mathrm{P} 505 \mathrm{~L}}$ mutation to rescue the growth and phenotypic defects in the mif2-3 TS mutants at the non-permissive temperature lends direct evidence for PfCENP-C functional dimerization domain in mediating its functions.

Thus, the disruption of the PfCENP-C dimerization domain results in the abolishment of kinetochore assembly and mitosis in yeast, confirmed by its detrimental effects on the mitotic spindles, chromosome segregation and the cell viability, providing evidence that the mutations in the conserved residues of PfCENP-C dimerization domain might also disrupt its dimer formation which in turn affects its high-order 
chromatin organization at the centromeres and renders the chromosome segregation machinery impaired.

It has been proposed that the loss of spindle integrity in the MIF2p-deficient cells is due to the defective spindle elongation during the anaphase but not the failure in spindle formation [34]. CENP-C has also been shown to maintain the kinetochore size as the impaired CENP-C functions lead to reduced diameter of kinetochores that may weaken or loosen the binding to the mitotic spindles [13]. Borrowing these concepts from the studies of Brown et al. and Tomkiel et al., the plausible explanation for the aberrant and disintegrated mitotic spindles in the mif2-3 and mif2-2 cells expressing the PfCENP-C N-terminal region, PfCENP-C motif and the PfCENP-C dimerization domain mutants (PfCENP-C ${ }^{\mathrm{F} 1993 \mathrm{~A}}$, PfCENP-C $\mathrm{C}^{\mathrm{F} 1996 \mathrm{~A}}$ and $P f C E N P-C^{\mathrm{Y} 2069 \mathrm{~A}}$ ) may be due to the failure of PfCENP-C to recruit and interact with the proteins that generate the stable spindle tension during the sister chromatids separation. This imbalance in the tension thus results in the abrupt breakage of the mitotic spindles. Alternatively, the spindles may fall apart due to the impaired spindle elongation during anaphase, resulting in structurally defective spindles. However, the spindle defects in the TS mutants are overcome by the PfCENP-C dimerization domain demonstrating its role in the maintenance of the structure of mitotic spindles. This also supports the idea that the PfCENP-C dimerization domain plays an important role in the kinetochore assembly at the centromeres and establishes connection with the mitotic spindles to ensure accurate chromosome segregation. The enrichment of PfCENP-C on the centromeric regions of P. falciparum as compared to the non-centromeric regions and its interaction with PfCENH3 and microtubules further strengthens its centromeric function in the malaria parasite.

These findings thus offer insights into the structuralfunctional details of various PfCENP-C domains especially the dimerization domain and its role in formation of a functional centromere-kinetochore structure.

\section{Conclusions}

The two conserved domains of PfCENP-C, the PfCENP$\mathrm{C}$ motif and the PfCENP-C dimerization domain have been mapped by bio-informatic analysis. The functional complementation assays establish the putative PfCENP$\mathrm{C}$ as a true CENP-C homologue involved in chromosome segregation, stable mitotic spindle morphology and cell viability. The key residues within the PfCENP$\mathrm{C}$ dimerization domain essential for its in vivo functions as a centromere protein have also been identified. The binding of PfCENP-C to the P. falciparum centromeres and its in vivo interactions with PfCENH3 and mitotic spindles further strengthens its centromere-associated functions.

\section{Additional files}

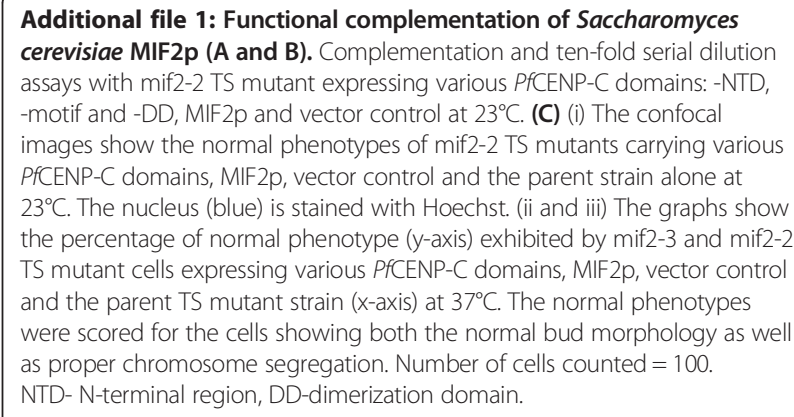

Additional file 2: Functionally essential residues within the PfCENPC dimerization domain. ( $A$ and $\mathbf{B}$ ) Growth and ten-fold serial dilution assays with the mif2-2 TS mutants expressing the PFCENP-C-DD mutants, PFCENP-C F1993A $^{\text {, PfCENP-C }}$ F1996A, PfCENP-C ${ }^{\text {Y2069A }}$ and PfCENP-C ${ }^{\mathrm{N} 2060 \mathrm{~A}}$ at $23^{\circ} \mathrm{C}$. All the constructs show normal growth at permissive temperature. (C) The phenotypes of mif2-2 TS mutants expressing PfCENP-CF1993A, PFCENP-C C $1996 \mathrm{~A}$, PFCENP-C ${ }^{\mathrm{Y} 2069 \mathrm{~A}}$ and PFCENP-C ${ }^{\mathrm{N} 2060 \mathrm{~A}}$ at $23^{\circ} \mathrm{C}$. The Hoechst stained nucleus (blue) shows proper chromosome segregation in all the constructs.

Additional file 3: The mitotic spindle integrity is maintained by the dimerization domain. (A) Confocal images showing the mitotic spindle (green) and the Hoechst stained nucleus (blue) of the mif2-2 TS mutant cells expressing the PFCENP-C domains: -NTD, -motif and -DD, MIF2 $p$ and empty vector alone at $23^{\circ} \mathrm{C}$. (B) The confocal images represent the mitotic spindles of mif2-2 TS mutants expressing PfCENP-C-DD mutants,

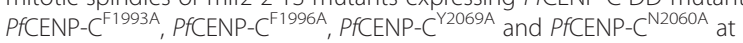
$23^{\circ} \mathrm{C}$. At permissive temperature, normal spindle morphology is observed by all the constructs expressed by mif2-2 TS mutants. The insets show the mitotic spindle structures.

\section{Abbreviations}

TS: Temperature sensitive; WT: Wild type; NTD: N-terminal region; DD: Dimerization domain.

\section{Competing interests}

The authors declare that they have no competing interests.

\section{Authors' contributions}

NS conceived and designed the experimental methods, contributed reagents for the experiments and corrected the manuscript. GV performed the experiments, analysed the data and wrote the manuscript. The final version of manuscript was approved by both the authors.

\section{Acknowledgements}

NS acknowledges DBT, Government of India for the award of TATA Innovation Fellowship. The authors thank Dr Charlie Boone and Ms Sondra Bahr, University of Toronto, Canada for providing the mif2-3 TS mutant (Y06477) and WT (DMA3865) S. cerevisiae strains. We sincerely acknowledge Dr Pamela B. Meluh, Johns Hopkins University School of Medicine, Baltimore, MD for sharing the mif2-2 TS mutant strain (6848-4-2). We also thank Ms B S Suma for her assistance in confocal microscopy. GV is a UGC Senior Research Fellow, Government of India.

Received: 22 May 2014 Accepted: 30 November 2014

Published: 4 December 2014

\section{References}

1. Earnshaw WC, Ratrie H III, Stetten G: Visualization of centromere proteins CENP-B and CENP-C on a stable dicentric chromosome in cytological spreads. Chromosoma 1989, 98:1-12.

2. Saitoh H, Tomkiel J, Cooke CA, Ratrie H III, Maurer M, Rothfield NF, Earnshaw WC: CENP-C, an autoantigen in scleroderma, is a component of the human inner kinetochore plate. Cell 1992, 70:115-125. 
3. Yang $\mathrm{CH}$, Tomkiel J, Saitoh $\mathrm{H}$, Johnson DH, Earnshaw WC: Identification of overlapping DNA-binding and centromere-targeting domains in the human kinetochore protein CENP-C. Mol Cell Biol 1996, 16:3576-3586.

4. Milks KJ, Moree B, Straight AF: Dissection of CENP-C-directed centromere and kinetochore assembly. Mol Biol Cell 2009, 20:4246-4255.

5. Tanaka K, Li Chang H, Kagami A, Watanabe Y: CENP-C functions as a scaffold for effectors with essential kinetochore functions in mitosis and meiosis. Dev Cell 2009, 17:334-343.

6. Cohen RL, Espelin CW, De Wulf P, Sorger PK, Harrison SC, Simons KT: Structural and functional dissection of Mif2p, a conserved DNA-binding kinetochore protein. Mol Biol Cell 2008, 19:4480-4491.

7. Carroll CW, Silva MC, Godek KM, Jansen LE, Straight AF: Centromere assembly requires the direct recognition of CENP-A nucleosomes by CENP-N. Nat Cell Biol 2009, 11:896-902.

8. Carroll CW, Milks KJ, Straight AF: Dual recognition of CENP-A nucleosomes is required for centromere assembly. J Cell Biol 2010, 189:1143-1155.

9. Guse A, Carroll CW, Moree B, Fuller CJ, Straight AF: In vitro centromere and kinetochore assembly on defined chromatin templates. Nature 2011, 477:354-358.

10. Moree B, Meyer CB, Fuller CJ, Straight AF: CENP-C recruits M18BP1 to centromeres to promote CENP-A chromatin assembly. J Cell Biol 2011, 194:855-871.

11. Fachinetti $D$, Folco HD, Nechemia-Arbely $Y$, Valente $L P$, Nguyen $K$, Wong $A J$ Cleveland DW: A two-step mechanism for epigenetic specification of centromere identity and function. Nat Cell Biol 2013, 9:1056-1066.

12. Kato H, Jiang J, Zhou BR, Rozendaal M, Feng H, Ghirlando R, Bai Y: A conserved mechanism for centromeric nucleosome recognition by centromere protein CENP-C. Science 2013, 340:1110-1113.

13. Tomkiel J, Cooke CA, Saitoh H, Bernat RL, Earnshaw WC: CENP-C is required for maintaining proper kinetochore size and for a timely transition to anaphase. J Cell Biol 1994, 125:531-545.

14. Sullivan BA, Schwartz S: Identification of centromeric antigens in dicentric Robertsonian translocations: CENP-C and CENP-E are necessary components of functional centromeres. Hum Mol Genet 1995, 4:2189-2197.

15. Knehr M, Poppe M, Schroeter D, Eickelbaum W, Finze EM, Kiesewetter UL, Paweletz N: Cellular expression of human centromere protein C demonstrates a cyclic behavior with highest abundance in the $\mathrm{G} 1$ phase. Proc Natl Acad Sci U S A 1996, 93:10234-10239.

16. Dawe RK, Reed LM, Yu HG, Muszynski MG, Hiatt EN: A maize homolog of mammalian CENPC is a constitutive component of the inner kinetochore. Plant Cell 1999, 11:1227-1238.

17. Song K, Gronemeyer B, Lu W, Eugster E, Tomkiel JE: Mutational analysis of the central centromere targeting domain of human centromere protein C, (CENP-C). Exp Cell Res 2002, 275:81-91.

18. Kwon MS, Hori T, Okada M, Fukagawa T: CENP-C is involved in chromosome segregation, mitotic checkpoint function, and kinetochore assembly. Mol Biol Cell 2007, 18:2155-2168.

19. Orr B, Sunkel CE: Drosophila CENP-C is essential for centromere identity. Chromosoma 2011, 120:83-96.

20. Przewloka MR, Venkei Z, Bolanos-Garcia VM, Debski J, Dadlez M, Glover DM: CENP-C is a structural platform for kinetochore assembly. Curr Biol 2011, 21:399-405.

21. Brown MT, Goetsch L, Hartwell LH: MIF2 is required for mitotic spindle integrity during anaphase spindle elongation in Saccharomyces cerevisiae. J Cell Biol 1993, 123:387-403.

22. Fukagawa T, Brown WR: Efficient conditional mutation of the vertebrate CENP-C gene. Hum Mol Genet 1997, 6:2301-2308.

23. Kalitsis P, Fowler KJ, Earle E, Hill J, Choo KA: Targeted disruption of mouse centromere protein $C$ gene leads to mitotic disarray and early embryo death. Proc Natl Acad Sci U S A 1998, 95:1136-1141.

24. Fukagawa T, Pendon C, Morris J, Brown W: CENP-C is necessary but not sufficient to induce formation of a functional centromere. EMBO J 1999, 18:4196-4209.

25. Fukagawa $T$, Regnier $V$, Ikemura $T$ : Creation and characterization of temperature-sensitive CENP-C mutants in vertebrate cells. Nucleic Acids Res 2001, 29:3796-3803.

26. Moore LL, Roth MB: Hcp-4, a Cenp-C-like protein in Caenorhabditis elegans, is required for resolution of sister centromeres. J Cell Biol 2001, 153:1199-1208.

27. Sugimoto K, Kuriyama K, Shibata A, Himeno M: Characterization of internal DNA-binding and C-terminal dimerization domains of human centromere/kinetochore autoantigen CENP-C in vitro: role of DNAbinding and self-associating activities in kinetochore organization. Chromosome Res 1997, 5:132-141.

28. Trazzi S, Bernardoni R, Diolaiti D, Politi V, Earnshaw WC, Perini G, Della Valle G. In vivo functional dissection of human inner kinetochore protein CENP-C. J Struct Biol 2002, 140:39-48.

29. Trazzi S, Perini G, Bernardoni R, Zoli M, Reese JC, Musacchio A, Della Valle G: The C-terminal domain of CENP-C displays multiple and critical functions for mammalian centromere formation. PLOS ONE 2009, 4:e5832.

30. Lanini L, McKeon F: Domains required for CENP-C assembly at the kinetochore. Mol Biol Cell 1995, 6:1049-1059.

31. Screpanti E, De Antoni A, Alushin GM, Petrovic A, Melis T, Nogales E, Musacchio A: Direct binding of Cenp-C to the Mis12 complex joins the inner and outer kinetochore. Curr Biol 2011, 21:391-398.

32. Sugimoto $\mathrm{K}$, Yata H, Muro Y, Himeno M: Human centromere protein C (CENP-C) is a DNA-binding protein which possesses a novel DNA-binding motif. J Biochem 1994, 116:877-881.

33. Politi V, Perini G, Trazzi S, Pliss A, Raska I, Earnshaw WC, Della Valle G: CENP-C binds the alpha-satellite DNA in vivo at specific centromere domains. J Cell Sci 2002, 115:2317-2327.

34. Brown MT: Sequence similarities between the yeast chromosome segregation protein Mif2 and the mammalian centromere protein CENP-C. Gene 1995, 160:111-116.

35. Talbert PB, Bryson TD, Henikoff S: Adaptive evolution of centromere proteins in plants and animals. J Biol 2004, 3:18.

36. Meluh PB, Koshland D: Evidence that the MIF2 gene of Saccharomyces cerevisiae encodes a centromere protein with homology to the mammalian centromere protein CENP-C. Mol Biol Cell 1995, 6:793-807.

37. Heeger S, Leismann O, Schittenhelm R, Schraidt O, Heidmann S, Lehner CF: Genetic interactions of separase regulatory subunits reveal the diverged Drosophila Cenp-C homolog. Genes Dev 2005, 19:2041-2053.

38. Meeks-Wagner D, Wood JS, Garvik B, Hartwell LH: Isolation of two genes that affect mitotic chromosome transmission in S. cerevisiae. Cell 1986, 44:53-63.

39. Verma G, Surolia N: Plasmodium falciparum CENH3 is able to functionally complement Cse4p and its, C-terminus is essential for centromere function. Mol Biochem Parasitol 2013, 192:21-29.

40. Hoeijmakers WA, Flueck C, Françoijs KJ, Smits AH, Wetzel J, Volz JC, Bártfai R: Plasmodium falciparum centromeres display a unique epigenetic makeup and cluster prior to and during schizogony. Cell Microbiol 2012, 14:1391-1401

41. Trager W, Jensen JB: Human malaria parasites in continuous culture. Science 1976, 193:673-675.

42. Chris L, Vanderberg JP: Synchronization of Plasmodium falciparum erythrocytic stages in culture. J Parasito/ 1979, 65:418-420.

43. Altschul SF, Gish W, Miller W, Myers EW, Lipman DJ: Basic local alignment search tool. J Mol Biol 1990, 215:403-410.

44. The Plasmodium Genome Resource: [http://PlasmoDB.org]

45. Harsha $\mathrm{HC}$, Molina H, Pandey A: Quantitative proteomics using stable isotope labeling with amino acids in cell culture. Nat Protoc 2008, 65:505-516.

46. Kruger NJ: The Bradford Method for Protein Quantitation. In Basic Protein and Peptide Protocols. Methods Mol Biol. Volume 32. Edited by Walker JM. Totowa, NJ: Humana Press Inc; 1994:9-15.

47. Burke D, Dawson D, Stearns T: Methods in Yeast Genetics: A Cold Spring Harbor Laboratory Course Manual. Cold Spring Harbor, New York: Cold Spring Harbor Laboratory Press; 2000.

48. Tonkin CJ, van Dooren GG, Spurck TP, Struck NS, Good RT, Handman E, McFadden Gl: Localization of organellar proteins in Plasmodium falciparum using a novel set of transfection vectors and a new immunofluorescence fixation method. Mol Biochem Parasitol 2004, 137:13-21.

49. Freitas-Junior LH, Hernandez-Rivas R, Ralph SA, Montiel-Condado D, Ruvalcaba-Salazar OK, Rojas-Meza AP, Scherf A: Telomeric heterochromatin propagation and histone acetylation control mutually exclusive expression of antigenic variation genes in malaria parasites. Cell 2005, 121:25-36.

50. Lopez-Rubio JJ, Gontijo AM, Nunes MC, Issar N, Hernandez Rivas R, Scherf A: $5^{\prime}$ flanking region of var genes nucleate histone modification patterns linked to phenotypic inheritance of virulence traits in malaria parasites. Mol Microbiol 2007, 66:1296-1305. 
51. Burkin DJ, Jones C, Burkin HR, McGrew JA, Broad TE: Sheep CENPB and CENPC genes show a high level of sequence similarity and conserved synteny with their human homologs. Cytogenet Cell Genet 1996, 74:86-89.

52. Warburton PE, Cooke CA, Bourassa S, Vafa O, Sullivan BA, Stetten G, Earnshaw WC: Immunolocalization of CENP-A suggests a distinct nucleosome structure at the inner kinetochore plate of active centromeres. Curr Biol 1997, 7:901-904.

53. Van Hooser AA, Ouspenski II, Gregson HC, Star DA, Yen TJ, Goldberg ML, Brinkley BR: Specification of kinetochore-forming chromatin by the histone H3 variant CENP-A. J Cell Sci 2001, 114:3529-3542.

doi:10.1186/1475-2875-13-475

Cite this article as: Verma and Surolia: The dimerization domain of

$P F C E N P-C$ is required for its functions as a centromere protein in human malaria parasite Plasmodium falciparum. Malaria Journal 2014 13:475.

\section{Submit your next manuscript to BioMed Central and take full advantage of:}

- Convenient online submission

- Thorough peer review

- No space constraints or color figure charges

- Immediate publication on acceptance

- Inclusion in PubMed, CAS, Scopus and Google Scholar

- Research which is freely available for redistribution 